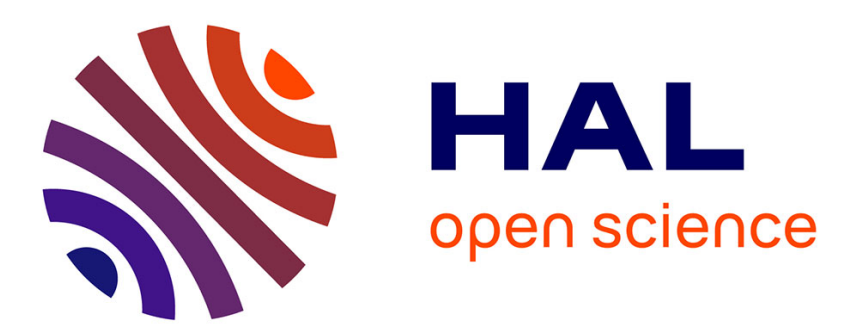

\title{
Le Jugement dernier de Fossa: une vision eschatologique intégrant la représentation du jugement immédiat
}

Marcello Angheben

\section{To cite this version:}

Marcello Angheben. Le Jugement dernier de Fossa : une vision eschatologique intégrant la représentation du jugement immédiat. Hortus artium medievalium: Journal of the International Research Center for Late Antiquity and Middle Ages, 2015, 21, pp.406-420. 10.1484/J.HAM.5.107403 . halshs02272911

\section{HAL Id: halshs-02272911 https://shs.hal.science/halshs-02272911}

Submitted on 30 Aug 2019

HAL is a multi-disciplinary open access archive for the deposit and dissemination of scientific research documents, whether they are published or not. The documents may come from teaching and research institutions in France or abroad, or from public or private research centers.
L'archive ouverte pluridisciplinaire HAL, est destinée au dépôt et à la diffusion de documents scientifiques de niveau recherche, publiés ou non, émanant des établissements d'enseignement et de recherche français ou étrangers, des laboratoires publics ou privés. 


\title{
LE JUGEMENT DERNIER DE FOSSA : UNE VISION ESCHATOLOGIQUE INTÉGRANT LA REPRÉSENTATION DU JUGEMENT IMMÉDIAT
}

\section{MARCELLO ANGHEBEN}

UDC: $726.59(450.65)$

75.046.3(450.65) "12"

Original scientific paper

Manuscript received: 26. 01. 2015.

Revised manuscript accepted: 14. 03. 2015.

DOI: 10.1484/J.HAM.5.107403
M. Angheben

Université de Poitiers

Centre d'études supérieures de civilisation médiévale

Poitiers, France

The Last Judgment, painted between 1260 and 1280 on the west wall of Santa Maria ad Cryptas in Fossa, Abruzzo, partly reproduces the classical Byzantine formula by developing on the first register a representation of the immediate judgment, the particular judgement following death, and the Waiting Paradise where the just await the final resurrection. This hypothesis can be based on two strong arguments. On the one hand, the weighing of souls and this paradise embodied by the bosom of the patriarchs were represented in a similar iconographic and stylistic way in the paintings at Bominaco, where the temporal nature of the judgment is indicated by the presence of the death of the rich man. On the other hand, two souls gathered in the bosom of the patriarchs seem to be moving away, as though preparing to unite with their body at the resurrection and join the kingdom of heaven. This reading of the paintings at Fossa in terms of double judgment may also be applied to the paintings at Pomposa and may be considered for those at Sant'Angelo in Formis and Sommacampagna.

Keywords: Fossa, Bominaco, Sant'Angelo in Formis, Pomposa, Sommacampagna, Last Judgment, particular judgment, Paradise, Bosom of Abraham, weighing of souls, scale, Parable of the Rich Man and Lazarus.

L'église de Santa Maria ad Cryptas de Fossa, située dans la province de L'Aquila, possède un vaste ensemble de peintures datées, pour la plupart, des années 1260-1280'. Comme le voulait une tradition très solidement ancrée, toute la surface

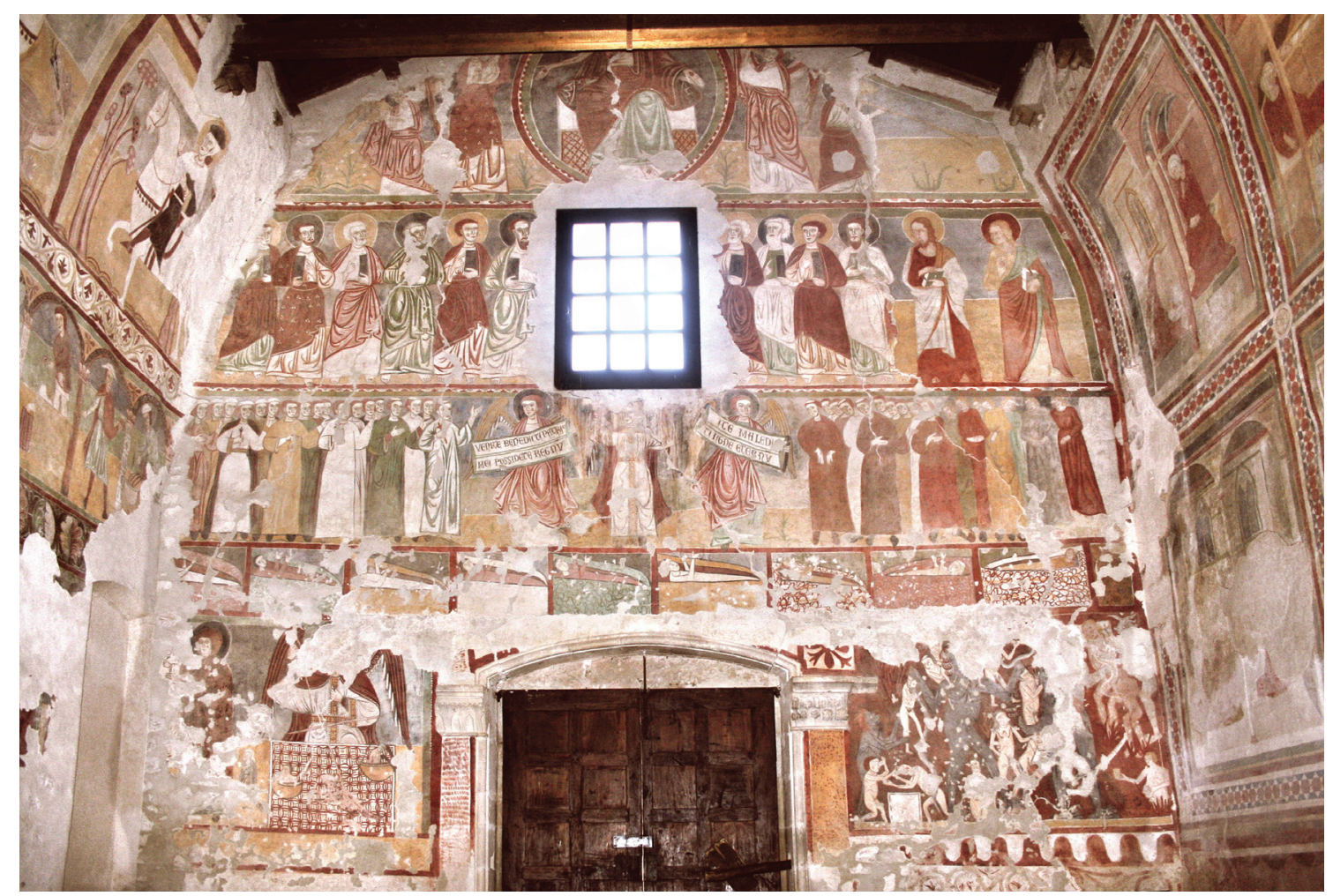

fig. 1 Fossa, Santa Maria ad Cryptas, contre-façade, le Jugement dernier (photo M. Angheben).

\begin{abstract}
${ }^{1}$ Les peintures de Fossa sont généralement situées entre celles de Bominaco (1263) et celle du tableau de Gentile della Rocca figurant la Vierge allaitant datée de 1283 : A(nno) D(omini) MCC. Octogesimo. III. Gentile d(e). Rocca. me. pi(nxit). Cf. É. BERTAUX, L'art dans l'Italie méridionale. De la fin de l'Empire Romain à la Conquête de Charles d'Anjou, Paris et Rome, 1968 [1 1 ìre éd. 1903], I, p. 298 (qui situe les peintures de Fossa quelques années après celles de Bominaco) ; G. MATTHIAE, Pittura medioevale abruzzese, Milan, 1969, pp. 59-62 ; V. PACE, Aggiornamento delle pp. 279-281 e 283-30o del capitolo : L'influence de l'école du Mont-Cassin, in L'art dans l'Italie méridionale. Aggiornamento dell'opera di Émile Bertaux sotto la direzione di Adriano Prandi, Rome, 1978, pp. 491-517, en part. p. 514 ; V. LUCHERINI, Pittura tardoduecentesca in Abruzzo. Gli affreschi di Fossa e l'attività della bottega di Gentile da Rocca, in Dialoghi di Storia dell'Arte, 8/9, 1999, pp. 80-89; M. DELLA VALLE, Osservazioni sui cicli pittorici di San Pellegrino a Bominaco e di Santa Maria ad Cryptas di Fossa in Abruzzo, in Acme, LIX, 3, 2006, pp. 101-158 ; S. PAONE et A. TOMEI, La pittura medievale nell'Abruzzo aquilano, Cinisello Balsamo, 2010, pp. 50-55. Le Jugement dernier n'a heureusement pas subi de destruction lors du tremblement de terre du 6 avril 2009, à l'inverse d'un des deux cava-
\end{abstract}






fig. 2 Bominaco, San Pellegrino, paroi nord, la mort du mauvais riche et l'enfer (photo M. Angheben).

de sa contre-façade a été consacrée à un Jugement dernier (fig. 1 $)^{2}$. Sa structure et ses composantes découlent à la fois de la formule byzantine classique et d'une formule occidentale dont la version la plus proche, y compris sur le plan géographique, est celle de Sant'Angelo in Formis. Comme sur la contre-façade de cette célèbre église campanienne de la fin du $\mathrm{XI}^{\text {e }}$ siècle, le Jugement dernier de Fossa se déploie sur cinq registres (fig. 5).

Au sommet, le Christ-Juge exprime son verdict en tournant sa main droite vers le haut et sa main gauche vers le bas. Il est accompagné sur ce même registre de quatre anges buccinateurs et, sur le registre sous-jacent, par les douze apôtres ${ }^{3}$. Ceux-ci se tiennent debout, et non sur un synthronon, et désignent pour la plupart le Christ, comme dans l'iconographie de l'Ascension ${ }^{4}$. Le registre médian est celui de la séparation : au centre, trois anges exposent des phylactères déroulés, comme à Sant'Angelo in Formis. Le phylactère de l'ange situé dans l'axe a malheureusement disparu, mais les deux autres comportent encore les sentences d'élection et de damnation de Matthieu 25 : «Venite benedicti patris mei possidete regnum » et «Ite maledicti in ignem eternum » (Mt 25, 34 et 41). De part et d'autre de ces anges se déploient le cortège des élus à dextre et celui des damnés à senestre. Quant à la résurrection, elle s'étend sur toute la largeur du deuxième registre où les morts sortent de leur tombeau au son des trompettes 5 .

Jusque là, cette composition ne se distingue guère des Jugements derniers italiens contemporains ou antérieurs. Il en va tout autrement pour le registre inférieur. Si l'enfer demeure relativement classique, la moitié de la paroi située à dextre accueille de manière tout à fait exceptionnelle la pesée des âmes, une petite femme non nimbée et une grande figure féminine nimbée, peut-être la Vierge. Mais le plus important est que ce registre se prolonge relativement loin sur la paroi méridionale de la nef où une porte monumentale ouvre sur un jardin occupé par le sein des patriarches (fig. 10-12). Dans les Jugements derniers byzantins, la présence du sein d'Abraham au milieu du jardin paradisiaque permet d'interpréter ce lieu non pas comme le séjour définitif dans lequel se rendent les élus après la résurrection, mais comme le paradis d'attente, celui où vont les âmes séparées de leur corps après la mort et le jugement particulier, que

liers situés sur la paroi sud, à côté de la contre-façade. Voir à ce sujet la notice de V. PACE (Fossa, S. Maria ad cryptas) dans Il terremoto del 6 aprile zoog in Abruzzo : danni, interventi, iniziative e schede, in Kunstchronik, 63, 2010, pp. 57-58. Pour l'iconographie du Jugement dernier de Fossa, voir G. RASETTI, Il Giudizio Universale in arte e la pittura medioevale abruzzese, Pescara, 1935, pp. 115-118; et J. BASCHET, Les justices de l'au-delà. Les représentations de l'enfer en France et en Italie (XII ${ }^{e}$-XV'siècle), Rome, 1993, pp. 205-206.

${ }^{2}$ Pour la localisation des Jugements derniers, voir P.K. KLEIN, L'emplacement du Jugement dernier et de la Seconde Parousie dans l'art monumental du haut Moyen Age, in L'emplacement et la fonction des images dans la peinture murale du Moyen Âge, Saint-Savin, 1992 (Actes du $5^{\mathrm{e}}$ séminaire International d'Art Mural, 16-18 septembre 1992, Saint-Savin), pp. 89-101. La signification qu'il accorde à cette localisation demeure cependant très discutable.

${ }_{3}^{3}$ L'extrémité droite de la paroi a manifestement subi des infiltrations car les surfaces endommagées ont été repeintes au-delà d'une ligne clairement visible. Ces repeints concernent surtout les deux apôtres de droite et les derniers damnés du registre inférieur.

${ }^{4}$ G. MATTHIAE, Pittura medioevale..., p. 51, a affirmé contre toute vraisemblance que les apôtres étaient assis.

${ }^{5}$ Le décalage entre la résurrection et les anges buccinateurs est d'autant plus étonnant quà Sant'Angelo in Formis, les deux thèmes ont été regroupés au registre supérieur, au-dessus du tribunal divin. On relèvera cependant qu’à Fossa, le registre supérieur est plus étroit que les autres et n’aurait dès lors pas fourni suffisamment de place pour la résurrection. 


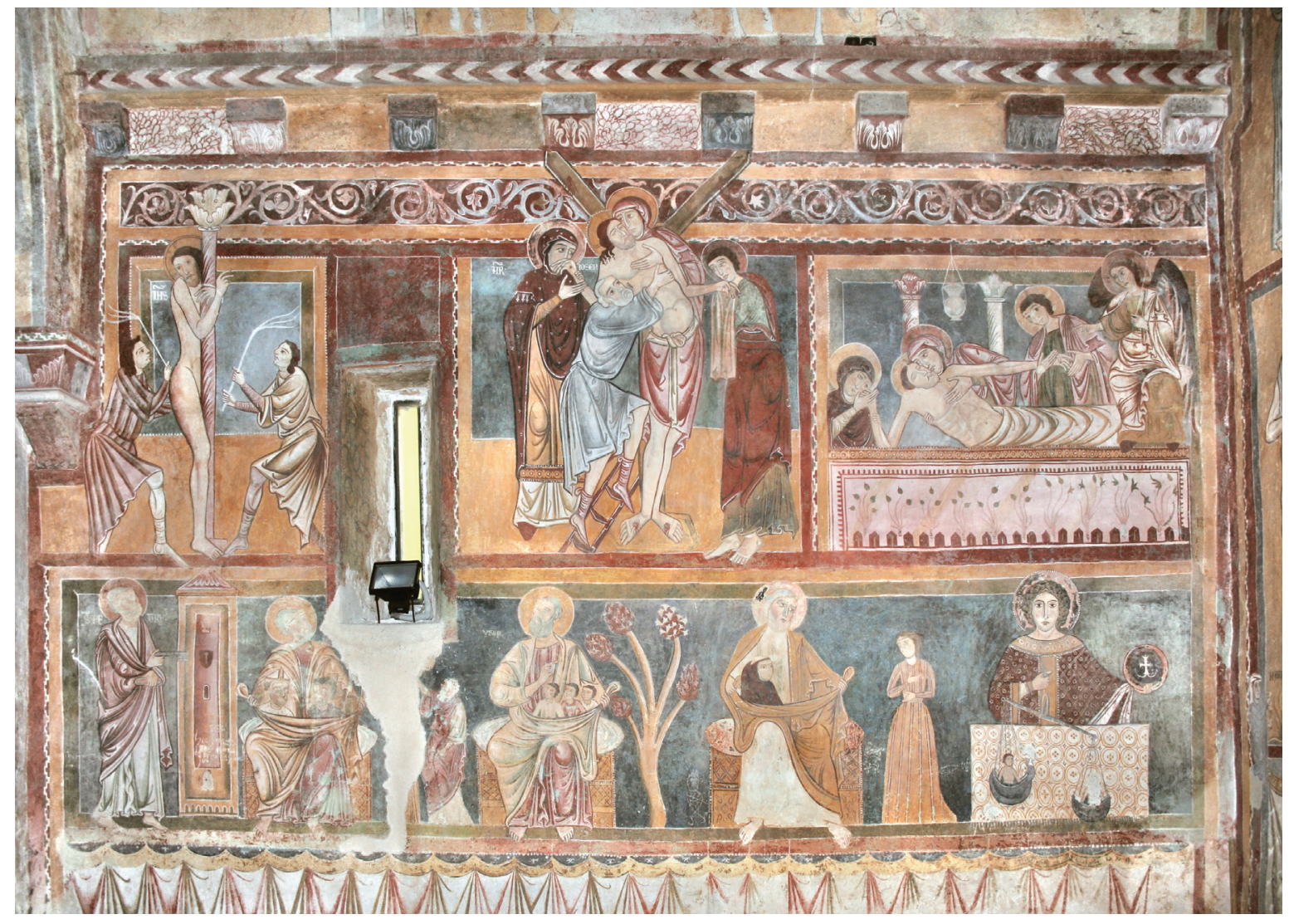

fig. 3 Bominaco, San Pellegrino, paroi sud, le jugement immédiat (photo M. Angheben).

je préfère appeler jugement immédiat, pour y attendre le Jugement dernier ${ }^{6}$. C'est ce que je pense avoir démontré dans une étude antérieure ${ }^{7}$.

Au départ de cette étude, je me suis demandé si cette hypothèse pouvait s'appliquer aux Jugements derniers italiens. Celui de Sant'Angelo in Formis ne comporte pas de représentation du sein d'Abraham et les autres composantes n'indiquent pas clairement si le concepteur a repris la signification présumée de la formule byzantine. Celui de Fossa intègre au contraire une représentation des âmes dans le sein des trois patriarches et d'autres indices confirmant leur interprétation en termes de paradis d'attente.

Un des indices les plus importants émane non pas du programme peint de Fossa mais de celui de la petite église de San Pellegrino de Bominaco dont les peintures, exécutées vers 1263 , présentent des formules iconographiques très semblables à celles de Fossa (fig. 2-3). De manière éminemment significative, ce programme comporte les thèmes visibles sur le premier registre de Fossa mais pas ceux des registres supérieurs : sur la paroi septentrionale se déploie en effet l'enfer, intégré ici dans le récit de la mort du mauvais riche, et sur la paroi méridionale se succèdent saint Pierre, une porte monumentale, un jardin abritant les trois patriarches et leurs protégés, un homme et une femme dépourvus de nimbe et la pesée des âmes ${ }^{8}$. La représentation de la mort du mauvais riche dans son lit situe très clairement les scènes de Bominaco dans le temps de la mort ${ }^{9}$. Dans la mesure où ce programme est antérieur à celui de Fossa, on peut supposer que les deux ensembles ont utilisé un modèle commun comportant les deux jugements et que le concepteur des peintures de Bominaco a isolé du Jugement dernier la représentation du jugement immédiat et du paradis d'attente pour en faire un usage original, sans doute adapté à une commande spécifique. Mais on pourrait également conjecturer que l'association de thèmes deutéroparousiaques à la composition de Bominaco émane du

\footnotetext{
${ }^{6}$ L'expression « jugement particulier » suggère en effet que ce jugement ne concerne qu'une âme à la fois alors que, dans les textes comme dans l'iconographie, il prend souvent un caractère collectif. Pour la question du jugement immédiat et de sa représentation, voir notamment J. BASCHET, Jugement de l'âme, Jugement dernier : contradiction, complémentarité, chevauchement? , in Revue Mabillon, n.s., 6, 1995, pp. 159-203 ; et M. ANGHEBEN, D’un Jugement à l'autre. La représentation du jugement immédiat dans les Jugements derniers français : 1100-1250, Turnhout, 2013, pp. 11-126.

${ }^{7}$ M. ANGHEBEN, Les Jugements derniers byzantins des XI'-XII e siècles et l'iconographie du paradis d'attente, in Cahiers archéologiques, 50, 2002, pp. 105134. J'ai également soutenu l'hypothèse du double jugement pour plusieurs Jugements derniers français dans M. ANGHEBEN, L'iconographie du portail de l'ancienne cathédrale de Mâcon : une vision synchronique du jugement individuel et du Jugement dernier, in Les cahiers de Saint-Michel de Cuxa, 32, 2001, pp. 73-87, et ID., D'un Jugement à l'autre..., pp. 165-541.

${ }^{8}$ Ces peintures sont datées avec précision de 1263, cf. É. BERTAUX, L’art dans l'Italie méridionale..., p. 290 ; G. MATTHIAE, Pittura medioevale..., pp. 31-44 ; V. PACE, Precisazioni sugli affreschi dell'oratorio di San Pellegrino a Bominaco, in Commentari, 21/4, 1970, pp. 291-297, en part. p. 291 ; V. LUCHERINI, Una proposta "romana" per gli affreschi duecenteschi di San Pellegrino a Bominaco (L’Aquila), in Napoli nobilissima, $5^{\mathrm{e}}$ ser., I, 5/6, 2000, pp. 163-188; et S. PAONE et A. TOMEI, La pittura..., pp. 35-5o. G. RASETTI, Il Giudizio Universale..., pp. 118-121 ; et J. BASCHET, Lieu sacré, lieu d'images. Les fresques de Bominaco (Abruzzes, 1263) : thèmes, parcours, fonctions, Paris, 1991, pp. 69-80, qui a donné la meilleure analyse iconographique de ces fresques, ont situé ces scènes dans le temps de la mort. En revanche, pour É. BERTAUX, L'art dans l'Italie méridionale..., p. 292, il s'agit d'un Jugement dernier.

${ }^{9}$ Le mauvais riche, identifié par une inscription, figure une deuxième fois dans l'enfer où il accomplit le geste traditionnel du doigt désignant sa bouche pour signifier qu'il demande à Abraham d'envoyer le pauvre Lazare tremper dans l'eau le bout de son doigt et rafraîchir sa langue (Lc 16, 24).
} 


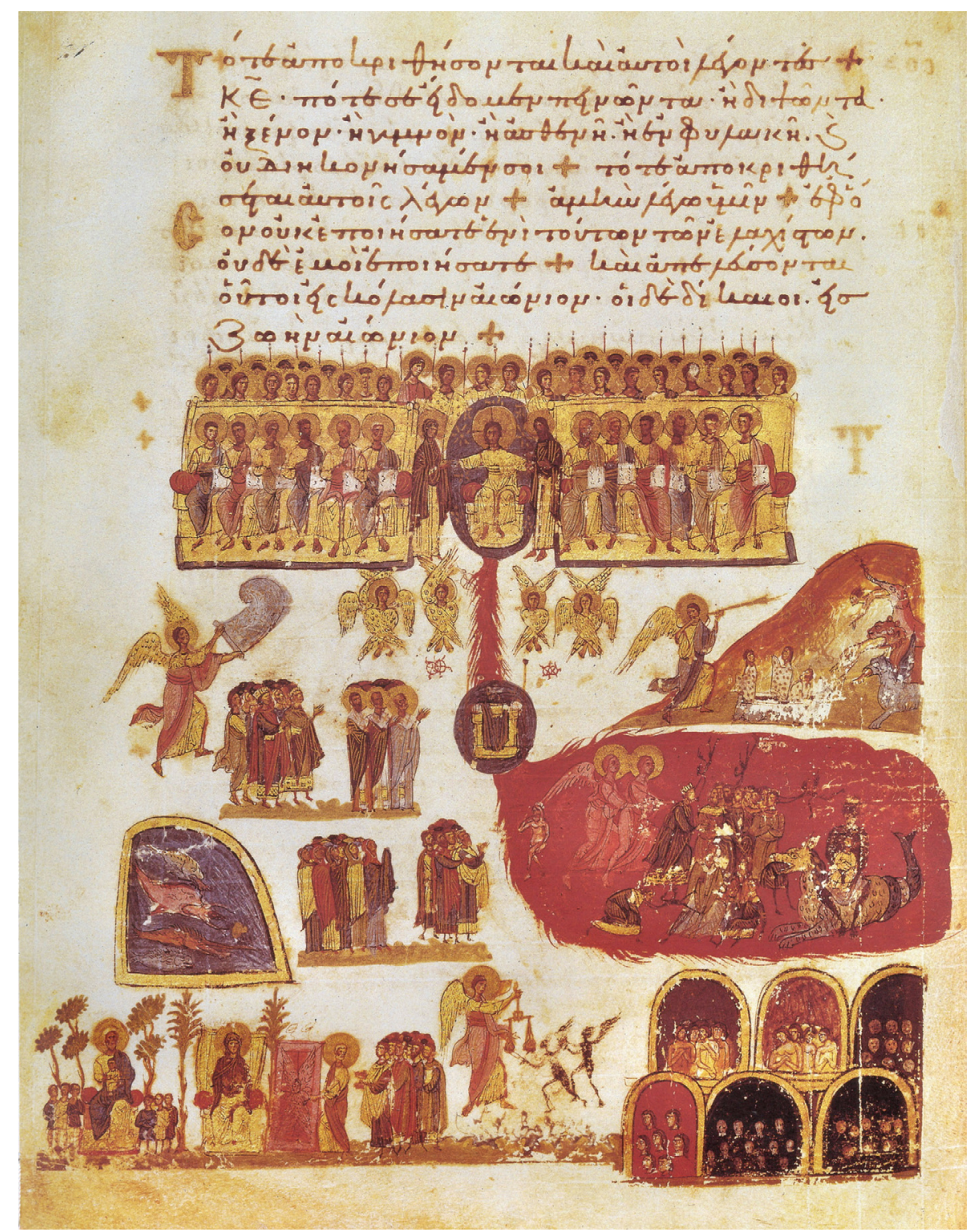

fig. 4 Trétraévangile de Stoudios, Paris BnF, ms. gr. 74, fo $51 v$; le Jugement dernier.

concepteur de Fossa ${ }^{10}$. L'essentiel reste que, dans ces deux hypothèses, la pesée de Bominaco s'effectue dans le cadre du jugement immédiat.

Les thèmes paradisiaques de Bominaco et de Fossa ont également été peints dans le bras gauche du transept de Santa Maria de Ronzano à Castelcastagna, dans un style analogue à celui de Fossa ${ }^{11}$. Ces peintures ne conservent cependant pas de représentation de l'enfer, de la mort du mauvais riche ou de thèmes spécifiques au Jugement dernier, de sorte que ni l'hypothèse du jugement immédiat ni celle du double jugement ne peut lui être appliquée avec certitude.

Pour pouvoir interpréter le premier registre de Fossa comme un jugement immédiat, il faudra rappeler très brièvement les arguments permettant de voir dans la formule byzantine classique une vision synchronique des deux jugements et préciser ensuite les raisons pour lesquelles cette hypothèse ne peut pas être transposée avec certitude aux peintures de Sant'Angelo in Formis. Pourront alors être abordés séparément les thèmes regroupés dans la moitié dextre du premier registre de Fossa : la pesée, l'élue conduite par une sainte, le jardin, le sein des patriarches et les élus recueillis dans ce lieu paradisiaque.

\section{LA FORMULE BYZANTINE CLASSIQUE}

L'œuvre reflétant le mieux le prototype de la formule byzantine classique est certainement le Tétraévangile de Stoudios, un manuscrit constantinopolitain de la seconde moitié du XI ${ }^{\mathrm{e}}$ siècle (fig. 4) ${ }^{12}$. Les mosaïques de Torcello,

\footnotetext{
10 É. BERTAUX, L'art dans l'Italie méridionale..., p. 299, a supposé l'existence d'un modèle commun pour la représentation des travaux des mois.

${ }^{n}$ V. PACE, Su Santa Maria di Ronzano : problemi e proposte, in Commentari, 20/4, 1969, pp. 259-269 ; V. LUCHERINI, Pittura tardoduecentesca..., pp. 8283 ; S. PAONE et A. TOMEI, La pittura..., pp. 54-55. Pour G. RASETTI, Il Giudizio Universale..., pp. 111-113, ces peintures ne se rattachent pas à un Jugement dernier disparu mais aux peintures de l'abside.

${ }^{12}$ S. TSUJI, The Headpiece Miniatures and Genealogy Pictures in Paris. gr. 74, in Dumbarton Oaks Papers, 29, 1975, pp. 166-203, en part. p. 170, a daté ce manuscrit entre 1060 et 1080 .

${ }^{13}$ I. ANDREESCU TREADGOLD, Torcello. III. La chronologie relative des mosaïques pariétales, in Dumbaton Oaks Papers, 30, 1976, pp. 247-341 ; EAD., The mosaics of Venice and the Venetian Lagoon. Thirty-five years of research at Torcello, in Arte medievale, 4 e sér., 3, 2013, Pp. 193-206 ; R. POLACCO, La cattedrale di Torcello, Venise et Trévise, 1984 ; et G. CAPUTO et G. GENTILI, Torcello alle origini di Venezia tra Occidente e Oriente, Venise, 2009.
} 
exécutées au XI ${ }^{\mathrm{e}}$ siècle ${ }^{13}$, et un ivoire du Victoria and Albert Museum, une œuvre italo-byzantine du XII ${ }^{\mathrm{e}}$ siècle, en sont également très proches ${ }^{14}$. Les icônes $n^{\circ} 150$ et 151 du Sinaï, datées respectivement du XI ${ }^{\mathrm{e}}$ et du XII ${ }^{\mathrm{e}}$ siècle s'en éloignent en revanche déjà considérablement et les autres occurrences des $\mathrm{XI}^{\mathrm{e}}-\mathrm{XII}{ }^{\mathrm{e}}$ siècles demeurent généralement incomplètes ${ }^{15}$. Le Tétraévangile de Stoudios montre au sommet le tribunal divin, sur les deux registres médians la résurrection, des groupes d'élus ressuscités et l'étang de feu dans lequel sont plongés les damnés, et sur le premier registre la pesée, les cavernes infernales et le jardin paradisiaque. L'analyse structurelle et thématique de cette composition a montré que le registre inférieur a été consacré au jugement immédiat et que seuls les thèmes des trois registres supérieurs concernent le Jugement dernier.

En Orient d'abord et en Occident plus tard, les textes ont corrélé l'usage de la balance non pas au Jugement dernier mais à celui qui succède à la mort. Le thème apparaît dès le VII ${ }^{\mathrm{e}}$ siècle dans un épisode de la Vie de saint Jean l'Aumônier dans lequel des démons empilent dans les plateaux d'une balance les mauvaises actions d'un avare jusqu'au moment où un ange vient le sauver en y déposant un simple pain, l'unique don concédé par ce pécheur ${ }^{16}$.

En fonction du verdict de la balance, les damnés et les élus sont envoyés dans des séjours temporaires dans lesquels ils attendent la résurrection finale. Les damnés entrent dans les cavernes infernales par une anfractuosité rocheuse à peine visible de laquelle surgissent des flammes et sont ensuite dénudés, décapités ou encore réduits à l'état de squelettes. À la fin des temps, ces mêmes damnés devront en revanche recouvrer leur intégrité corporelle avant d'être plongés dans l'étang de feu en même temps que l'enfer. C'est exactement ce que montrent les deux registres supérieurs : à la résurrection, des damnés ayant récupéré leur statut socioprofessionnel sont poussés dans cet étang par le haut et non plus par l'antre enflammé du registre inférieur.

Quant aux élus, ils se dirigent vers un lieu paradisiaque que sa végétation et surtout sa porte défendue par un chérubin assimilent clairement à l'Éden. L'emplacement, la nature et les occupants de ce paradis le désignent plus précisément comme un séjour paradisiaque temporaire. Pour commencer, il se trouve très fortement marginalisé par sa position inférieure et latérale. Il est donc très éloigné du Christ et du ciel alors que les élus devraient pouvoir contempler leur Créateur, comme le font les ressuscités des registres médians. Les élus du registre inférieur doivent au contraire s'éloigner de lui en s'engageant dans un cortège divergent et en lui tournant le dos.

L'Éden correspond par ailleurs au séjour temporaire des âmes séparées. Les textes ne s'accordent pas toujours sur ce point, confondant parfois le paradis d'Adam avec le royaume des cieux. Les conceptions les plus cohérentes, comme celle de Moïse Bar Képha (avant 903), établissent toutefois une distinction très nette entre les deux ${ }^{17}$.

Les occupants sont également caractéristiques de ce séjour : la Vierge, le bon larron, absent du Tétraévangile de Stoudios mais présent dans la quasi-totalité des autres versions, et surtout le sein d'Abraham. La présence de la Vierge dans cette sorte d'Éden céleste se fonde sur plusieurs textes, à commencer par la Dormition du Pseudo-Jean dont certaines versions précisent que son corps a été déposé sous l'arbre de $v^{1}{ }^{18}$. L'idée est également présente dans l'Apocalypse de la $V_{\text {ierge }}{ }^{19}$. C'est également ce que suggère une enluminure d'un manuscrit des homélies de Jacques de Kokkinobaphos : dans un contexte temporel succédant à la Descente aux Limbes figurée sur les deux registres supérieurs, la Vierge trônant au milieu du paradis et flanquée de deux anges reçoit les prières d'Adam et d'Ėve prosternés à ses pieds et celles d'une foule encore plongée en enfer ${ }^{20}$. Avant la fin des temps, la Mère de Dieu semble donc bien destinée à séjourner dans l'Éden et à y exercer son pouvoir d'intercession.

Le deuxième occupant traditionnel du jardin paradisiaque est le bon larron. Alors qu'il se trouvait sur la croix, Jésus lui dit ceci : « Je te le déclare, en vérité, aujourd'hui, tu seras avec moi dans le paradis » (Lc 23, 43). Le bon larron a donc reçu le privilège d'entrer le premier au paradis, avant les justes de l'Ancien Testament extraits du Limbe des patriarches au moment de l'Anastasis. C'est également ce qu'affirment plus prolixement les Actes de Pilate, un apocryphe du IV $\mathrm{IV}^{\mathrm{e}}$ siècle ${ }^{21}$, et l'on en trouve des échos dans les icônes tardives et, en Occident, sur un émail de la « paix d'Aribert » : au-dessus de l'Anastasis et à droite de la Crucifixion, le Christ entraîne par la main le bon larron tandis que celui-ci porte sa croix sur l'épaule, comme dans les Jugements derniers byzantins ${ }^{22}$. Dans la mesure où le privilège reçu par le bon larron concerne les instants qui suivent la mort, sa

${ }_{14}$ Y. CHRISTE, Jugements derniers, Saint-Léger-Vauban, 1999, p. 27. B. BRENK, Tradition und Neuerung in der christlichen Kunst des ersten Jahrtausends. Studien zur Geschichte des Weltgerichtsbildes, Vienne, 1966 (Wiener byzantinische Studien, 3), p. 84, a situé cet ivoire au X' siècle. P. WILLIAMSON, Medieval Ivory Carvings. Early Christian to Romanesque, Londres, 2010, p. 129-130, l'a daté du XIe.

${ }^{15}$ G. et M. SÔTIRIOU, Icônes du Mont Sinaï, Athènes, 1958, II, pp. 128-131 ; et K. WEITZMANN, Die Ikone. 6. bis 14. Jahrhundert, Munich, 1978, p. 23.

${ }^{16}$ B. DE GAIFFIER, Pesée des âmes. À propos de la mort de l'empereur saint Henri II (1024), in Études critiques d'hagiographie et d'iconographie, Bruxelles, 1967, pp. 246-253.

${ }^{17}$ MOÏSE BAR KÉPHA, Commentaria in paradiso, P.G. 111, 485 C-D, 500 B-501 B et 494 D-495 B.

${ }^{18}$ C'est ce que l'on trouve notamment dans le Transitus grec « R», cf. S.C. MIMOUNI, Dormition et Assomption de Marie. Histoire des traditions anciennes, Paris, 1995 (Théologie historique), pp. 127-135, en part. p. 130.

${ }^{19}$ Apocalypse de la Vierge, 25, éd. M.R. JAMES, Apocrypha Anecdota. A Collection of Thirteen Apocryphal Books and Fragments, Cambridge, 1893, pp. 109126. Ce texte a été traduit en italien par M. ERBETTA, Gli apocrifi del Nuovo Testamento, Turin, 1975, III, pp. 447-454, en part. p. 452. Voir également S.C. MIMOUNI, Les Apocalypses de la Vierge. État de la question, in Apocrypha, 4, 1993, pp. 101-112.

${ }^{20}$ Homélies de Jacques de Kokkinobaphos, Bibl. du Vatican, cod. gr. 1162, fo . 48 v. Cette enluminure illustre le psaume 67, 7, cf. A.D. KARTSONIS, Anastasis. The Making of an Image, Princeton, 1986, p. 173.

${ }^{21}$ Actes de Pilate, 26, éd. H.C. KIM, The Gospel of Nicodemus. Gesta salvatoris, Toronto, 1973, pp. 46-47. Voir également W. SCHNEEMELCHER, New Testament Apocrypha, Cambridge et Louisville, I, 1991, pp. 501-534.

${ }^{22}$ G.A. VERGANI, "Omne regnum italicum ad suum disponebat nutum". Ariberto di Intimiano, vescovo guerriero e commitente, in Il Crocifisso di Ariberto. Un mistero millenario intorno al simbolo della cristianità, Milan, 1997, pp. 47-56, en part. pp. 52-53. Voir aussi dans cet ouvrage la notice n ${ }^{\circ} 6$ du catalogue, p. 188. 
présence dans le paradis des Jugements derniers byzantins ne se justifie pleinement que dans le contexte du jugement immédiat.

Le sein d'Abraham est encore plus caractéristique du paradis d'attente car, dans la parabole du pauvre Lazare, c'est dans ce lieu étrange que les anges conduisent l'âme de ce dernier immédiatement après sa mort. Comme pour l'Éden, les textes ne s'accordent pas toujours sur le statut du sein d'Abraham, mais les conceptions les plus cohérentes le désignent clairement comme le séjour dans lequel les élus attendent la résurrection. C'est le cas en particulier dans la liturgie constantinopolitaine ${ }^{23}$. L'iconographie du sein d'Abraham confirme par ailleurs cette lecture puisque les élus ont reçu l'apparence d'enfants et s'assimilent ainsi à des âmes séparées.

Dans le manuscrit de Stoudios, un indice déterminant confirme l'hypothèse des deux jugements : le cortège des élus accueilli par saint Pierre devant la porte du paradis ne comporte que des laïcs alors que des évêques se tiennent à la tête des groupes d'élus ressuscités des registres médians et sont de surcroît les seuls élus gratifiés d'un nimbe. Cela signifie non pas que ces prélats seront privés de cette récompense, mais que ce sont au contraire des privilégiés qui ne doivent pas transiter par le paradis d'attente. Ce privilège est clairement affirmé dans une oraison de la liturgie funéraire constantinopolitaine où les évêques ne sont pas appelés à se rendre dans le sein d'Abraham comme les autres défunts, mais dans un lieu céleste où ils verront le trône du Seigneur ${ }^{24}$. C'est exactement ce que montre l'iconographie : les évêques ne se dirigent pas vers le sein d'Abraham, ils sont les seuls à bénéficier d'un nimbe et sont les premiers à contempler le Christ. Il ne fait dès lors pratiquement aucun doute à mes yeux que le jardin du registre inférieur est destiné aux âmes séparées et que l'évaluation qui y donne accès est le jugement immédiat.

De manière extrêmement significative, les peintures cappadociennes de Karşi kilise (1212) montrent séparément les trois grands thèmes du registre inférieur de la formule classique : il en subsiste la porte du paradis et le bon larron à droite, la pesée au centre et l'enfer à gauche. En les détachant de la sorte des composantes spécifiques au Jugement dernier, on a donc clairement affirmé que ces thèmes possédaient une cohérence intrinsèque et, par conséquent, une grande autonomie ${ }^{25}$. C'est également ce que l'on a fait à Bominaco en représentant isolément la pesée, le paradis d'attente et l'enfer.

\section{SANT'ANGELO IN FORMIS}

$\mathrm{Au} \mathrm{XIV}^{\mathrm{e}}$ siècle et peut-être plus tôt, le premier registre des Jugements derniers byzantins a toutefois cessé de figurer

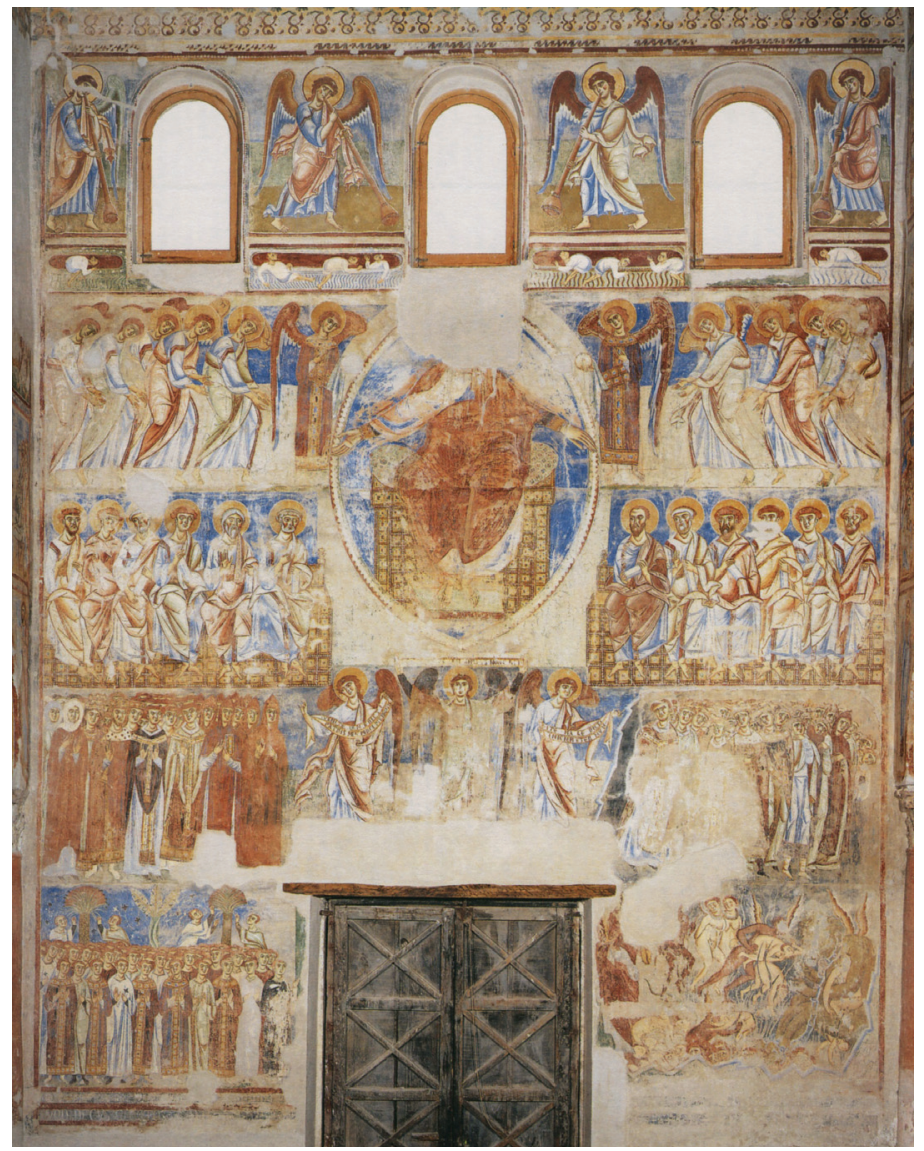

fig. 5 Sant'Angelo in Formis, contre-façade, le Jugement dernier, vue générale.

le jugement immédiat et son paradis verdoyant a été transformé en un paradis définitif ${ }^{26}$. On ne peut donc pas appliquer automatiquement l'hypothèse du double jugement à chaque occurrence de cette formule, en particulier lorsqu'elle se situe en Italie. Bien que le Jugement dernier de Torcello demeure le plus proche de celui de Stoudios, la présence d'une porte au centre du premier registre a fait disparaître le cortège des élus et laisse par conséquent subsister un doute sur l'interprétation du jardin paradisiaque.

La question se pose également pour les peintures de Sant'Angelo in Formis, même si elles ne dépendent pratiquement pas de la formule byzantine (fig. 5). L'espace situé sous le Christ est en effet occupé non pas par l'Hétimasie ni par le fleuve de feu mais par les anges déroulant les phylactères contentant les sentences d'élection et de damnation, comme sur les peintures arméniennes de Tat'ev, exécutées au $\mathrm{X}^{\mathrm{e}}$ siècle par des artistes occidentaux, dans l'Apocalypse de Bamberg et plus tard à Fossa ${ }^{27}$. Une structure proche de la formule byzantine apparaît toutefois du côté des élus : au deuxième registre, un cortège hiérarchisé se dirige vers l'ange portant la sentence

\footnotetext{
${ }^{23}$ M. ARRANZ, Le preghiere per i defunti nella tradizione bizantina. I sacramenti della restaurazione dell'antico Eucologio constantinopolitano, in Orientalia Christiana Periodica, 63, 1997, pp. 99-117. Le sein d’Abraham est mentionné dans les prières E1:3, ligne 10, E1:4, ligne 15 et E1:7, ligne 3.

${ }^{24}$ E2:3, cf. M. ARRANZ, Le preghiere per i defunti..., p. 112.

${ }^{25}$ C. JOLIVET-LÉVY, La Cappadoce médiévale, Paris, 2001, pp. 274-276, a également vu dans cette composition une représentation du jugement particulier. ${ }^{26}$ Ce sera le cas notamment sur les peintures de Kharie Djami, au XIVe siècle, et plus tard dans le Jugement dernier du réfectoire de Lavra au Mont-Athos où l'on voit les saints les plus éminents dotés d'un nimbe pénétrant dans le jardin paradisiaque. Mais cette lecture en termes de jugement unique pourrait également s'appliquer aux deux icônes du Sinaï sur lesquelles la résurrection des morts figure au même niveau que ce paradis.

${ }^{27}$ Les peintures de la cathédrale Saints-Pierre-et-Paul de Tat'ev à Siwnik, réalisées en 930 par des artistes occidentaux, permettent de faire remonter le thème des anges aux phylactères à l'époque carolingienne, autrement dit bien avant les manuscrits de Bamberg. Pour cette question et pour les origines du Jugement dernier de Sant'Angelo in Formis, je me permets de renvoyer à V. PACE et M. ANGHEBEN, Alfa e Omega, Il Giudizio Universale tra Oriente e Occidente X-XIV Secolo, Milan et Castel Bolognese, 2006, p. 36 et pp. 62-63. Pour Sant'Angelo in Formis, voir également J. WETTSTEIN, Sant' Angelo
} 


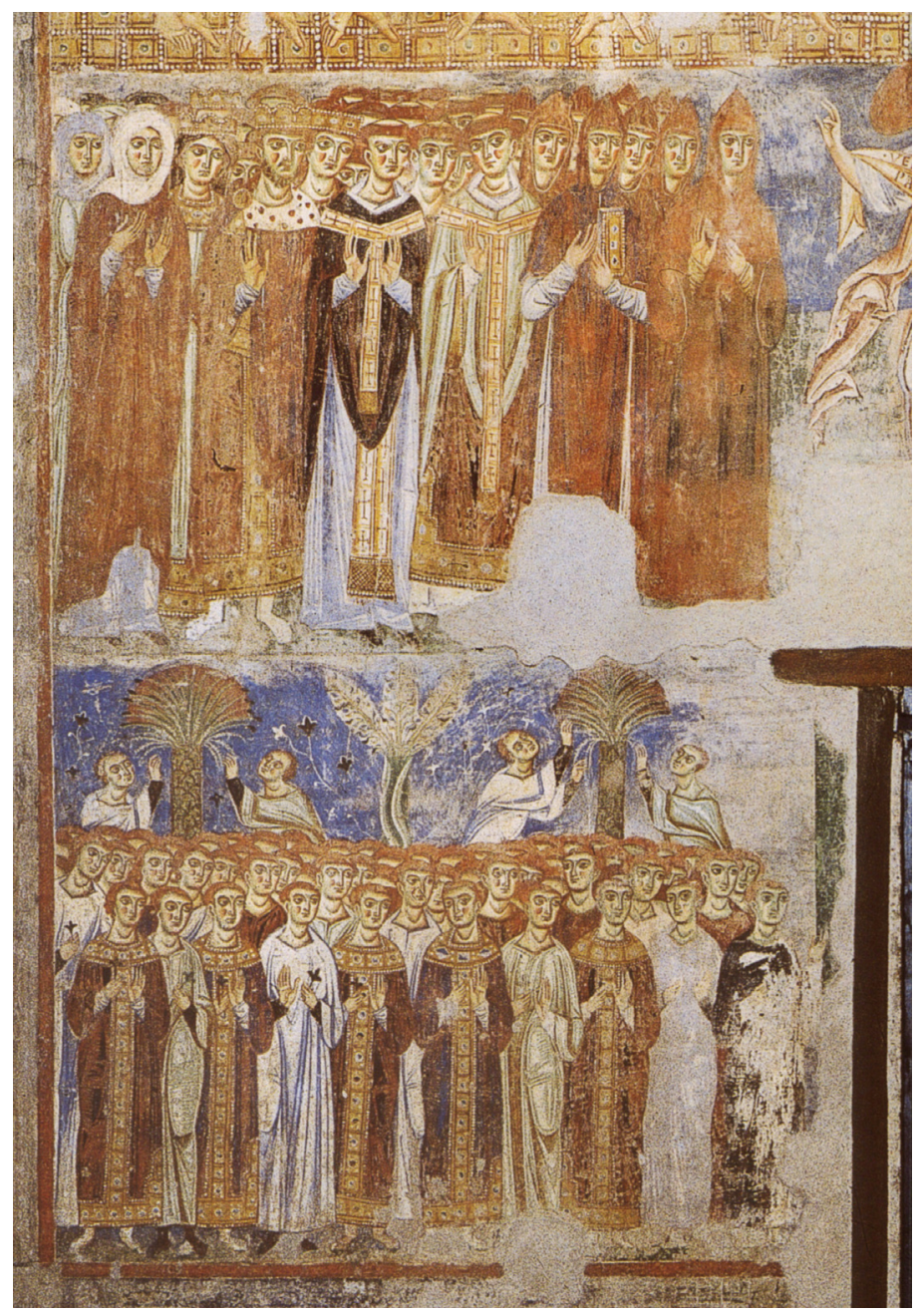

fig. 6 Sant'Angelo in Formis, contre-façade, le paradis.

d'élection tandis quau registre inférieur, des élus anonymes se tiennent immobiles dans un jardin paradisiaque (fig. 6). Ces derniers possèdent une taille nettement réduite par rapport aux élus du deuxième registre, ce sont apparemment tous des hommes et ils portent, au premier plan, alternativement une simple tunique blanche et un vêtement somptueux orné d'orfrois et, au second plan, des tuniques blanches ou rouges. À l'arrière plan enfin, quatre élus tendent une main vers un palmier, probablement pour en cueillir les fruits.

La nature édénique de ce séjour, la taille et l'anonymat des élus suggèrent que ceux-ci sont des âmes séparées alors que ceux du deuxième registre sont des corps réunis à leur âme ${ }^{28}$. Quant aux différents types de vêtements, ils correspondraient à leurs mérites. L'hypothèse demeure cependant fragile car aucun de ces indices ne possède la force de ceux qui ont été énumérés au sujet de la formule byzantine. Il en va tout autrement à Fossa.

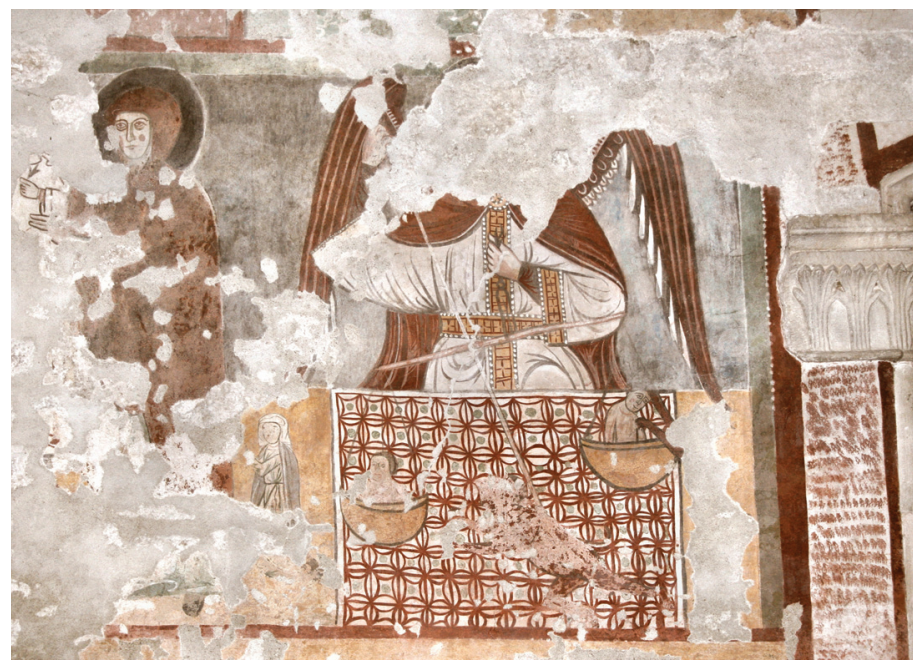

fig. 7 Fossa, Santa Maria ad Cryptas, contre-façade, la pesée des actions (photo M. Angheben).

\section{LES THÈMES DU PREMIER REGISTRE DE FOSSA}

Comme dans les Jugements derniers byzantins, le premier registre s'articule autour de la pesée, si ce n'est que la porte de la contre-façade a contraint le concepteurà déplacer ce thème dans la moitié dextre de la composition. À partir de ce jugement, les âmes sont supposées se rendre en enfer ou dans le sein des patriarches. L'enfer ne se rattache cependant pas à la scène de la pesée, pas plus quà la séparation du troisième registre. Cet enfer est de surcroît l'unique lieu de supplice de la composition, alors que la formule byzantine avait superposé les cavernes infernales et l'étang de feu pour bien distinguer les destinations des damnés avant et après la Parousie. Dans l'hypothèse du double jugement, on doit donc supposer que cet enfer est destiné à la fois aux âmes séparées et à celles qui ont été réunies à leur corps au moment de la résurrection.

\section{La pesée}

Dressé derrière une forme rectangulaire finement décorée, un ange, certainement saint Michel, tient une balance dont les plateaux portent chacun une petite figure humaine, tout en transperçant un diable de sa lance (fig. 7). De toute évidence, ce sont les âmes qui sont évaluées ici et non les actes. L'âme située à senestre est clairement celle d'un damné : elle s'inscrit du côté de l'enfer, elle présente un visage attristé et un corps affaissé, et un diable volontairement effacé l'agrippe fermement de ses deux pattes antérieures ${ }^{29}$. L'autre âme est en revanche celle d'un élu, même si son plateau est plus bas que celui du damné, car il se tourne vers le jardin paradisiaque et, surtout, saint Michel le défend

in Formis et la peinture médiévale en Campanie, Genève, 1960, pp. 60-61 ; O. MORISANI, Gli Affreschi di S. Angelo in Formis, Naples, 1962, pp. 53-55 ; A. MOPPERT-SCHMIDT, Die Fresken von S.Angelo in Formis, Zürich, 1967, pp. 93-103 ; C.I. MINOTT, The Iconography of the Frescoes of the Life of Christ in the Church of Sant'Angelo in Formis, PhD, Princeton University, 1967, pp. 207-228 ; J. WETTSTEIN, La fresque romane. Études comparatives, I. Italie France - Espagne, Paris, 1971, pp. 71-73 ; G. GUNHOUSE, The Fresco decoration of Sant'Angelo in Formis, Ph.D., The Johns Hopkins University, 1992, pp. 153-157 ; G.M. JACOBITTI et S. ABITA, La basilica benedettina di Sant’Angelo in Formis, Naples, 1992, p. 55 ; J. BASCHET, Les justices de l'au-delà..., pp. 198-202 ; et Y. CHRISTE, Jugements derniers..., p. 278.

${ }^{28}$ C.I. MINOTT, The Iconography..., p. 222, est l'un des rares auteurs à s'être interrogé sur la raison d'être de ce paradis inférieur, mais ses réponses ne sont guère satisfaisantes.

${ }_{29}$ Pour l'iconographie de la pesée et la problématique relative à son objet, voir M.P. PERRY, On the Psychostasis in Christian Art, in Burlington Magazine, 22, 1912-1913, pp. 94-105, et pp. 208-218 ; et L. KRETZENBACHER, Die Seelenwaage. Zur religiösen Idee vom Jenseitsgericht auf der Schicksalwaage in Hochreligion, Bildkunst und Volksglaube, Klagenfurt, 1958. 


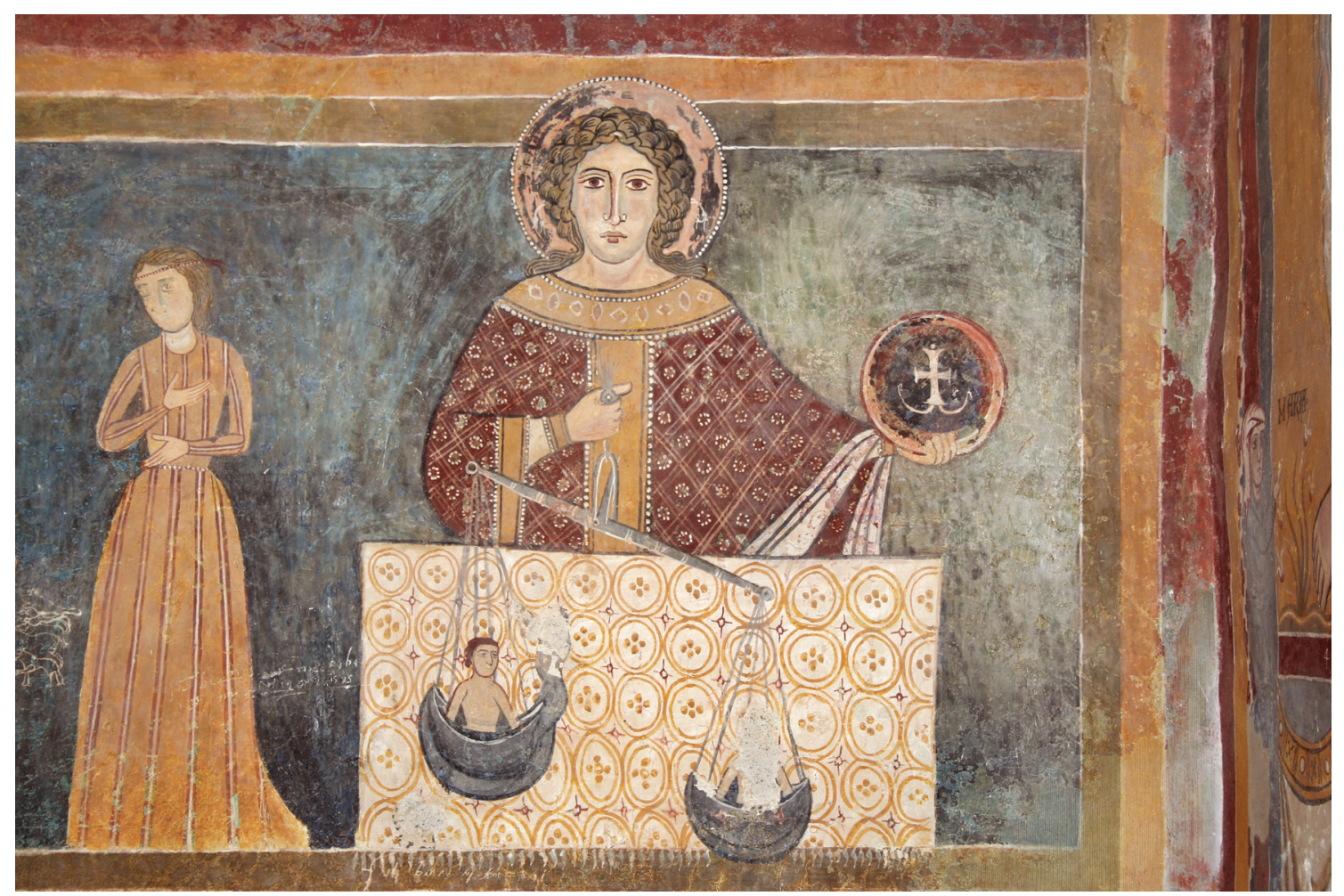

fig. 8 Bominaco, San Pellegrino, paroi sud, la pesée des actions (photo M. Angheben).

avec énergie contre le diable, également gratté, qui tente de s'emparer de lui.

L'objet rectangulaire derrière lequel se tiennent l'ange de Fossa et ceux de Bominaco et de Ronzano est probablement un autel car le thème figure également dans de nombreux Jugements derniers comme celui du tondo de la pinacothèque vaticane et surtout celui de l'évangéliaire de Wölfenbuttel où un calice figure à la fois sur la table d'autel et sur un plateau de la balance ${ }^{30}$. Les formes rectangulaires de Fossa, de Bominaco et de Ronzano sont dépourvues de calice ou de tout autre objet liturgique, mais l'ange aptère de Bominaco est vêtu d'une chasuble, semblable à celle que porte saint Magne dans une scène de la crypte d'Anagni $^{31}$. Dans cette perspective, l'objet circulaire qu'il exhibe dans la main gauche pourrait être interprété comme étant une hostie. En dépit de l'usure de la peinture, cet objet semble toutefois posséder du volume et pourrait dès lors correspondre plus classiquement à une sphère. Il me paraît difficile de trancher. Le vêtement liturgique de l'archange suffit en tout cas pour supposer qu'il se tient devant un autel et que celui-ci évoque les messes célébrées pour le salut des défunts ${ }^{32}$.

En Occident comme en Orient, les textes stipulent généralement que le jugement immédiat s'effectue au moyen d'une balance. Un des meilleurs exemples est fourni par la légende de l'empereur Henri II relatée dans la chronique de Léon d'Ostie et souvent représentée. Dans ce récit comme dans ses nombreuses transcriptions visuelles, saint Laurent sauve l'empereur en jetant dans un plateau de la balance le calice d'or que ce dernier avait offert à une église ${ }^{33}$. Le thème apparaît également dans plusieurs jugements immédiats dont la signification est attestée par la présence du lit de mort. C'est le cas notamment sur le portail de l'église de Perse, à Espalion, et à la façade de Saint-Pierre de Spolète où elle figure à la fois dans la mort du juste et dans celle de l'impie $^{34}$. Dans un manuscrit des Étymologies d'Isidore de Séville conservé à Prüfening (116o-1165), c'est le scribe luimême, le moine Swicher, qui s'est représenté sur son lit de

\footnotetext{
30 Wolfenbüttel, Herzog August Bibliothek, Cod. Guelf. 65 Helmst., fo 13 v. Pour le tondo du Vatican, voir R. SUCKALE, Die Weltgerichtstafel aus dem römischen Frauenkonvent S. Maria in Campo Marzio als programmatisches Bild der einsetzenden Gregorianischen Kirchenreform, in Das mittelalterliche Bild als Zeitzeuge. Sechs Studien, Berlin, 2002, pp. 12-122. On peut également citer les exemples de San Giovanni a Porta Latina, Santa Cecilia in Trastevere, Santa Maria in Vescovio et Santa Maria Donnaregina à Naples.

${ }^{31}$ L. CAPPELLETTI, Gli affreschi della cripta anagnina. Iconologia, Rome, 2002, fig. 82.

${ }^{32}$ Pour É. BERTAUX, L'art dans l'Italie méridionale..., p. 295, il s'agit d'une table et saint Michel porte un globe. Pour G. RASETTI, Il Giudizio Universale..., p. 112, les peintures de Ronzano, Fossa et Bominaco représentent une sorte de banc derrière lequel est assis saint Michel. J. BASCHET, Lieu sacré, lieu d'images..., pp. 76-78, a qualifié la forme rectangulaire de Bominaco de «plan spécifique sur lequel se déroule la pesée ». On notera que la pesée figure juste en dessous de la mise au tombeau. Aussi pourrait-on supposer que le concepteur a voulu établir une correspondance entre le tombeau et l'autel, mais les autres scènes de la Passion - Flagellation et Descente de Croix - ne correspondent guère aux sujets sous-jacents, respectivement saint Pierre devant la porte du paradis et le sein des patriarches.

33 J. ROOSVAL, Die Seelenwägung Kaiser Heinrichs II. in der gotländischen Malerei, in Zeitschrift für Kunstwissenschaft, 4, 1950, pp. 125-130 ; KRETZENBACHER, Die Seelenwaage..., p. 120 ; DE GAIFFIER, Pesée des âmes..., pp. 249-251 ; et R.W. SCHELLER, Die Seelenwägung und das Kelchwunder Kaiser Heinrichs II., Amsterdam, 1997, pp. 7-20.

${ }^{34}$ J. ESCH, La chiesa di San Pietro di Spoleto. La facciata et le sculture, Florence, 1981, pp. 101-106 ; J. BASCHET, Jugement de l'âme, Jugement dernier..., pp. 176-177 ; et M. ANGHEBEN, D'un Jugement à l'autre..., pp. 84-95.
} 


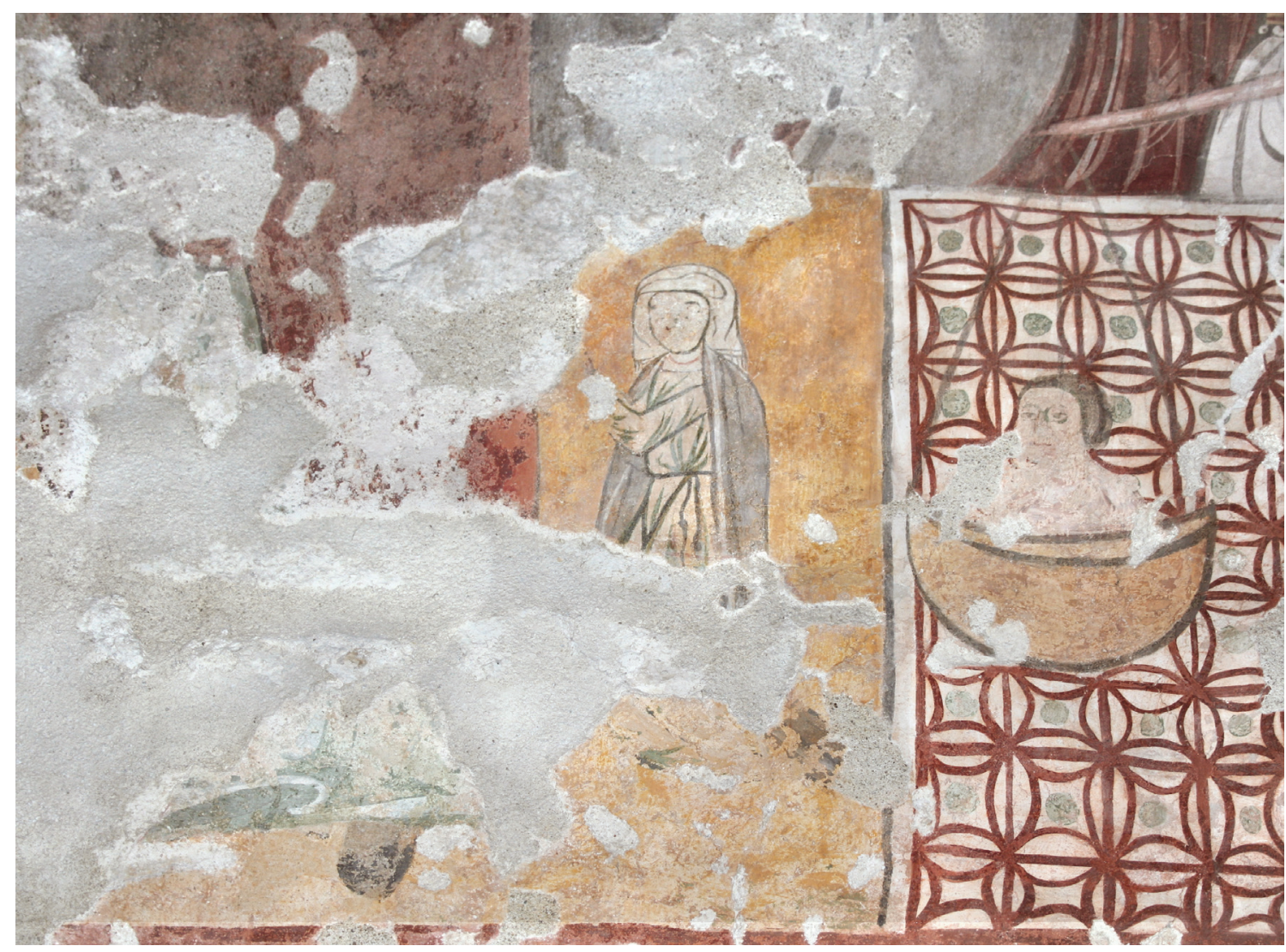

fig. 9 Fossa, Santa Maria ad Cryptas, contre-façade, une élue conduite par une sainte (photo M. Angheben).

mort et c'est un livre recopié de sa main posé sur un plateau de la balance qui assure son salut ${ }^{35}$.

La pesée de Fossa avait déjà été figurée avec quelques variantes à Bominaco (fig. 8). Pour commencer, saint Michel y est dépourvu d'ailes. Ensuite, la figure émergeant du plateau le plus haut se trouve du côté du paradis, ce qui paraît plus logique, et un diable tente de faire pencher ce plateau vers le bas, tandis que la figure peinte sur l'autre plateau a été grattée au même titre que ce diable. Si l'on admet que l'auteur de cette dégradation a bien compris le sens de l'image, on peut en déduire que son geste confirme le statut de damné affectéà ce personnage ${ }^{36}$. Quant à l'ange, il tient de la main gauche non plus une lance mais un objet sphérique ou circulaire marqué d'une croix déjà mentionné. Face à cette scène apparaît la mort du mauvais riche, issue de la parabole du pauvre Lazare. Comme dans les exemples précédents, le thème du transitus développé autour du lit de mort confirme que la pesée concerne les âmes des défunts.

À Fossa, cette hypothèse est corroborée par le dédoublement du jugement et de la séparation. Au troisième registre, les anges aux phylactères se chargent en effet de séparer les damnés et les élus. L'ostension des sentences est relativement fréquente dans les Jugements derniers occidentaux et elle constitue généralement l'unique mode de séparation, comme on peut le voir dans l'Apocalypse de Bamberg et à Sant'Angelo in Formis. À Fossa en revanche, on a ajouté, deux registres plus bas, une deuxième séparation opérée cette fois au moyen d'une balance et sans le moindre rapport visuel avec la première. Les élus du troisième registre désignent au contraire le haut, probablement le Christ et le ciel auquel ils sont destinés, et ne semblent dès lors pas appelés à descendre au premier registre pour y être évalués par la balance et à s'engager dans la direction opposée afin de pouvoir pénétrer dans le jardin paradisiaque ${ }^{37}$. L'explication la plus logique est, une fois encore, celle de la superposition des deux jugements.

\section{L'élue conduite par une sainte}

À côté de la pesée, une élue se tourne vers le jardin paradisiaque et progresse seule dans cette direction (fig. 9). En dépit des lacunes, on peut supposer que la grande figure nimbée est la Vierge ou une autre sainte guidant les pas de cette femme et répondant ainsi aux prières qu'elle lui a adressées de son vivant ou que lui destinent ses proches ${ }^{38}$. Sa taille, nettement réduite par rapport à celle de la Vierge

\footnotetext{
35 Munich, Bayerische Staatsbibliothek, Clm 13031, fo ${ }_{1}$ (Prüfening, 1160-1165), cf. E. KLEMM, Isidor von Sevilla, Etymologiae, in Ornamenta ecclesiae, Kunst und Künstler der Romanik, Cologne, 1985 (Katalog zur Ausstellung des Schnütgen-Museums in der Josef-Haubrich-Kunsthalle), I, p. 241 , fig. B39.

${ }^{36}$ Pour J. BASCHET, Lieu sacré, lieu d'images..., p. 77, au contraire, la figure de gauche représente les mauvaises actions. Il considère par ailleurs que la pesée ne concerne que l'élue figurée à gauche de la scène.

${ }^{37}$ Les damnés désignent un lieu situé derrière eux, probablement l'enfer, même si celui-ci figure deux registres plus bas. L'extrémité droite du registre a cependant été repeinte, de sorte qu'on ignore quels étaient les gestes des derniers damnés. Les ressuscités tournent en revanche tous les mains vers le haut et semblent sereins face au sort qui les attend. Il se pourrait dès lors que le concepteur ait limité la représentation de la résurrection des morts aux seuls élus, comme dans plusieurs Jugements derniers français : Mâcon, Conques, Chartres et Reims.

$3^{8}$ Pour J. BASCHET, Les justices de l'au-delà..., p. 206, cette sainte est bien la Vierge.
} 


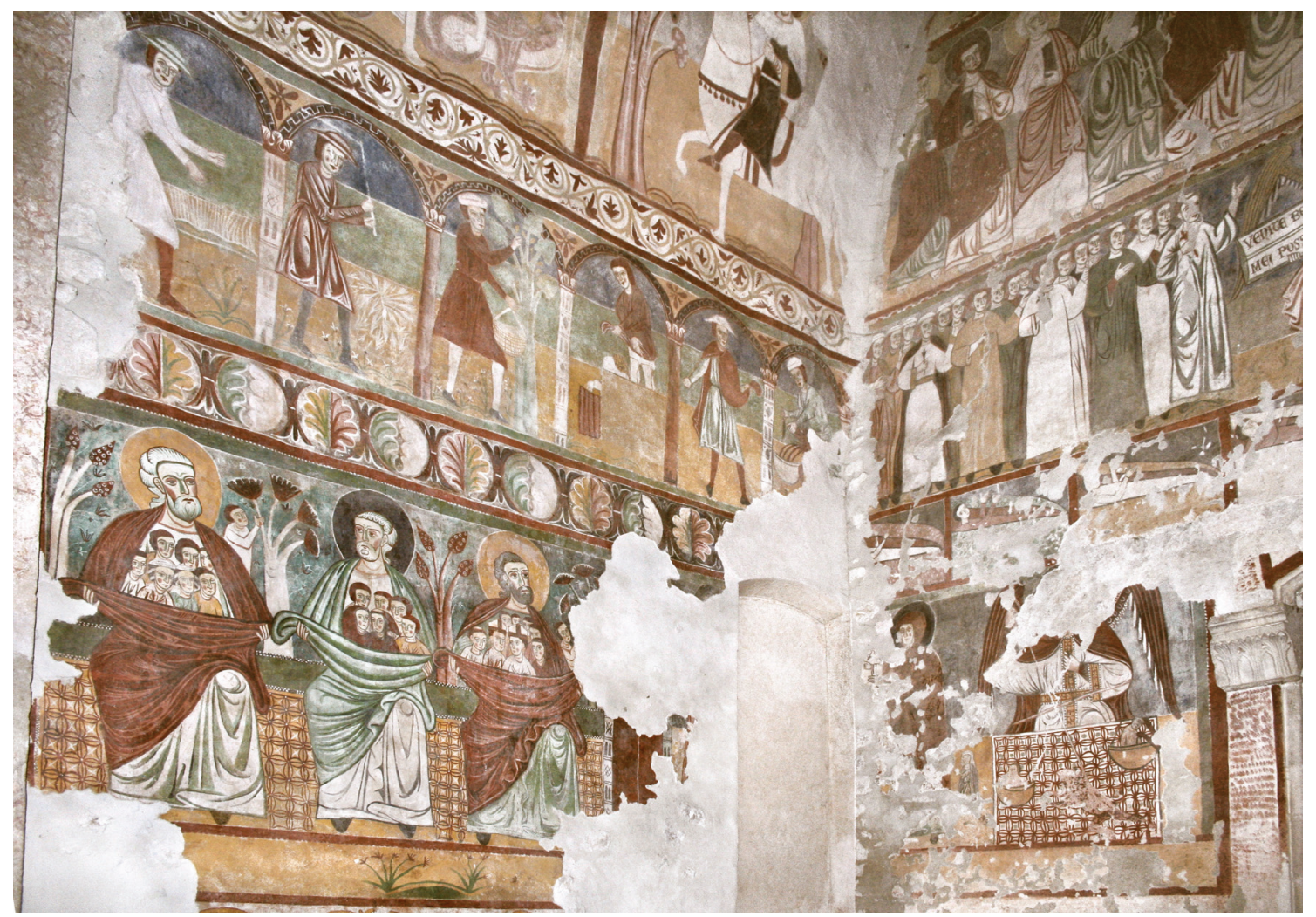

fig. 10 Fossa, Santa Maria ad Cryptas, paroi sud de la nef, le jugement immédiat et le paradis d'attente, vue générale (photo M. Angheben).

mais aussi par rapport aux élus ressuscités du troisième registre, suggère avec force qu'il s'agit d'une âme séparée. Son isolement confirme amplement ce statut. Les jugements immédiats peuvent en effet concerner une personne isolée ou des groupes d'élus intégrés dans un cortège, mais les Jugements derniers ne montrent jamais des élus solitaires, ce qui conduit à exclure cette possibilité.

Il est probable qu'un ou plusieurs autres élus ont figuré dans la lacune située entre l'angle de la paroi et la porte du paradis puisque quatre élus accompagnent saint Pierre dans la composition de Ronzano et que le paradis de Bominaco comporte une femme et un homme : après avoir franchi avec succès l'épreuve de la balance, cette femme replie les bras sur son corps et se dirige seule vers le sein des patriarches, sans être guidée par un saint. Plus loin, entre les deux patriarches de gauche, un homme tend son bras droit vers le haut, peutêtre pour cueillir le fruit d'un arbre. Il est probable dès lors que les peintures de Fossa ont également montré un ou plusieurs élus plus avancés dans leur progression vers le paradis. Il n'en demeure pas moins que l'élue a été isolée, suggérant ainsi que son âme a été jugée séparément.

Dans une telle configuration, cette femme et le ou les autres élus présumés pourraient correspondre à des commanditaires. Le programme du chœur montre les membres de la famille du donateur, Morel de Saours, agenouillés devant la Crucifixion qui occupe le centre de la paroi du chevet $^{39}$. On pourrait donc concevoir que cette famille a également financé les peintures de la nef et qu'en retour, elle a souhaité se voir représentée parmi les élus. C'est d'autant plus vraisemblable que Morel de Saours s'est également fait représenter avec sa famille dans l'église de Santo Spirito d'Ocre, à quelques kilomètres de Fossa, sous une Crucifixion et vraisemblablement dans le cadre d'un monument funéraire, ce qui attesterait une réelle préoccupation de celui qui fut peut-être le seigneur de Fossa pour le salut de son âme et des membres de sa famille ${ }^{40}$. L'élue du jugement immédiat porte toutefois des vêtements très différents de ceux qu'arborent les membres féminins de la famille de Morel et pourrait dès lors appartenir à une autre famille. Quoi qu'il en soit, on peut supposer que la figuration du premier jugement a servi à montrer l'efficacité des dons adressés à l'Église pour l'obtention du salut posthume, ainsi que le spécifient généralement les chartes de donations ${ }^{41}$.

\section{Le jardin paradisiaque}

Dans la zone de raccord malheureusement lacunaire située entre la pesée et le jardin paradisiaque, on devine

\footnotetext{
39 Pour l'iconographie et l'identité des commanditaires figurés dans le chœur, voir D. PICCIRILLI, Committenza francese in Abruzzo nella prima età angioina, in D. BENATI et A. TOMEI (éd.), L'Abruzzo in età angioina. Arte di frontiera tra Medioevo e Rinascimento. Atti del convegno, Chieti, 1-2 aprile 2004, Cinisello Balsamo, 2005, pp. 49-65.

$4^{40}$ D. PICCIRILLI, Committenza francese..., p. 49.

${ }^{41}$ Voir notamment, dans l'immense bibliographie concernant ce sujet, P. JOBERT, La notion de donation : convergences, 630-750, Paris, 1977, pp. 205-225 ; E. MAGNANI SOARES-CHRISTEN, Transforming Things and Persons. The Gifts pro anima in the Eleventh and Twelfth Centuries, in G. ALGAZI, V. GROEBNER et B. JUSSEN (éd.), Negotiating the Gift : Pre-Modern Figurations of Exange, Göttingen, 2003, pp. 269-284; EAD., Les médiévistes et le don. Avant et après la théorie maussienne, in E. MAGNANI (éd.), Don et sciences sociales. Théories et pratiques croisées, Dijon, 2007, pp. 15-28 ; et W. DAVIES, Buying with Masses. "Donation" pro remedio animae in Tenth-Century Galicia and Castile-León, in F. BOUGARD, C. LA ROCCA et R. LE JAN (dir.), Sauver son âme et se perpétuer : transmission du patrimoine et mémoire au haut Moyen Âge, Rome, 2005 (Actes de la table ronde "Salvarsi l'anima, perpetuare la famiglia » réunie à Padoue les 3, 4 et 5 octobre 2002), pp. 401-416.
} 


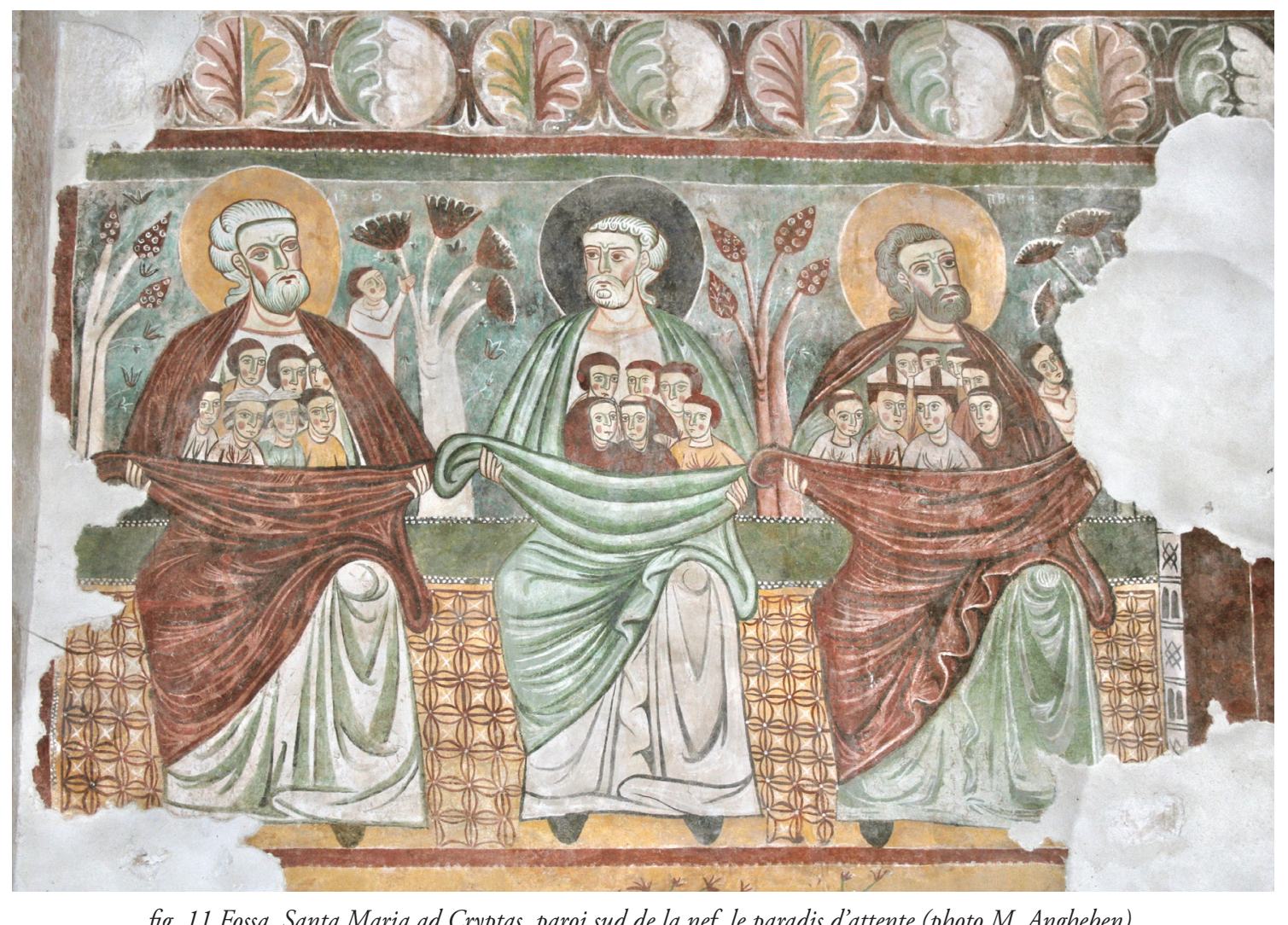

fig. 11 Fossa, Santa Maria ad Cryptas, paroi sud de la nef, le paradis d'attente (photo M. Angheben).

les formes du vêtement d'un personnage ainsi qu'une porte

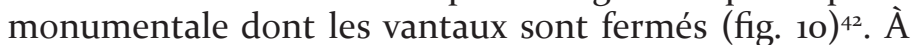
Bominaco et à Ronzano, saint Pierre se présente devant la porte du paradis et se prépare à introduire sa clé dans la serrure, ce qui laisse supposer que l'apôtre figurait également à Fossa, au même endroit ${ }^{43}$. Il faut tout de même relever quà Bominaco, la porte se trouve à l'extrémité gauche de la composition, sans la moindre relation avec la pesée, ce qui me semble difficilement explicable.

Derrière la porte de Fossa se déploient un jardin fleuri et arboré et, au premier plan, un synthronon accueillant les trois patriarches. Ce jardin s'assimile donc clairement à l'Éden, d'autant que les peintures de la même paroi de la nef montrent des scènes de la Création se déroulant dans un paysage identique ${ }^{44}$. Comme en Orient, les textes ont parfois confondu ou fusionné le paradis d'Adam avec la Jérusalem céleste ${ }^{45}$. Dans l'iconographie en revanche, les séjours édéniques sont généralement temporaires. On peut citer à cet égard plusieurs représentations du sein d'Abraham inscrites dans le récit de la parabole, comme dans le célèbre Codex Aureus d'Echternach ${ }^{46}$ et sur le portail de Moissac, ou dans le cadre de la destinée posthume des âmes comme dans le Nécrologe d'Obermünster ${ }^{47}$. La nature édénique du séjour des élus contribue dès lors, dans une mesure relativement modeste, à confirmer l'hypothèse du double jugement.

Il faut encore relever que par sa localisation sur la paroi de la nef, ce paradis est encore plus marginalisé que dans la formule byzantine. La structure exceptionnelle de ce Jugement accentue donc considérablement la distinction entre ce séjour temporaire et le royaume des cieux.

\section{Le sein des patriarches}

Mais, comme dans la formule byzantine, l'indice le plus déterminant émane du sein d'Abraham, auquel s'ajoute ici le sein des deux autres patriarches : Isaac et Jacob (fig. 11) ${ }^{48}$. Dans la liturgie funéraire comme dans la parabole, les âmes des élus sont clairement destinées au sein d'Abraham :

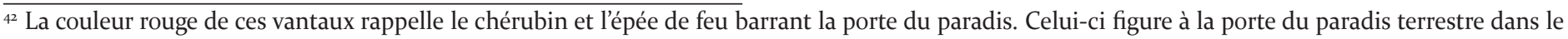
cycle du Péché originel de la paroi sud de la nef de Fossa mais apparemment pas devant le paradis d'attente.

43 V. LUCHERINI, Pittura tardoduecentesca..., p. 83, a également formulé cette hypothèse.

${ }^{44}$ Il est possible que le jardin paradisiaque ait été intentionnellement corrélé à l'Éden du cycle de la Création. Un lien pourrait également être envisagé avec les travaux des mois figurés juste au-dessus, mais cette fois dans une relation antithétique opposant la nature dans laquelle les hommes doivent travailler à celle, paradisiaque, dans laquelle séjournent les élus. Pour l'iconographie des mois à Fossa et Bominaco, voir V. BRANCONE, Complementi iconografici per il calendario dipinto dell'oratorio di San Pellegrino a Bominaco, in Arte medievale, III, 2, 2004, pp. 75-108.

${ }^{45}$ AUGUSTIN, De Genesi ad litteram libri duodecim, XII, 34, 65-67, éd. et trad. P. AGAËSSE et A. SOLIGNAC, Paris, 1972 (Bibliothèque augustinienne, 49), pp. 447-451. Voir également J. DELUMEAU, Une histoire du paradis. I. Le jardin des délices, 1992, p. 46 ; et J. BASCHET, Jugement de l'âme, Jugement dernier..., pp. 162-163.

${ }^{46}$ E. ROSENTHAL, Abraham and Lazarus. Iconographical Considerations of a Medieval Book Painting, in The Pacific Art Review, 4, 1945-1946, pp. 7-23.

${ }^{47}$ Munich, Haupstaatsarchiv, Obermünster, 1, fo 74v. (1177-1183), cf. J. BASCHET, Le sein du père. Abraham et la paternité dans l'Occident médiéval, Paris, 2000, p. 120, et pl. I.

${ }^{48}$ Il faut préciser que les trois patriarches sont évoqués conjointement dans l'Évangile de Matthieu parce que les élus s'attableront avec eux à la fin des temps (Mt 8, 11-12) et qu'ils ne sont donc supposés les accueillir en leur sein qu'après la mort.
} 


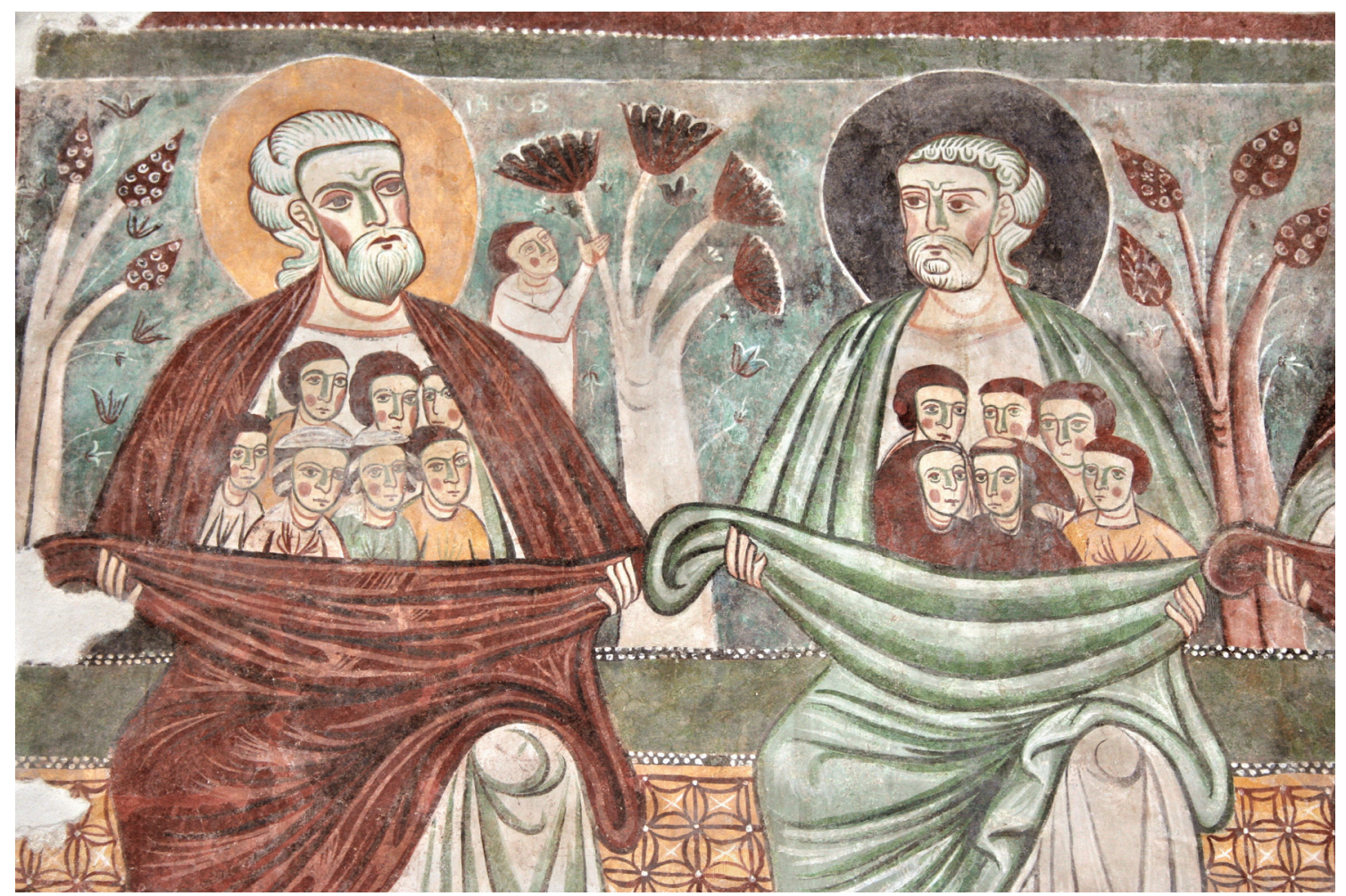

fig. 12 Fossa, Santa Maria ad Cryptas, paroi sud de la nef, le sein des patriarches, détail (photo M. Angheben).

«Que le chœur des anges t'enlève et te dépose dans le sein d'Abraham, puisses-tu jouir du repos éternel avec le pauvre Lazare $»^{49}$. Il en va de même dans l'iconographie. Un des exemples les plus remarquables est une enluminure du Nécrologe d'Obermünster car ce livre concerne les âmes des défunts commémorées à la date anniversaire de leur mort : Abraham se tient au milieu d'un paradis édénique caractérisé par la végétation déjà évoquée et davantage encore par les personnifications des quatre fleuves du paradis, tandis que des anges en vol apportent des âmes dans son sein.

En Occident comme en Orient, le sein d'Abraham est toutefois devenu un séjour définitif dès leXII ${ }^{\mathrm{e}}$ siècle et davantage encore au XIII siècle. C'est particulièrement frappant sur les mosaïques du baptistère de Florence où les élus sont appelés à rejoindre le sein des patriarches non pas à la suite de la pesée des âmes ou des actions mais au terme du jugement divin dont le verdict favorable transcrit sur un phylactère est ostensiblement exposé aux ressuscités ${ }^{\circ}$. À Fossa, la situation est très différente puisque le jugement a été dédoublé et que le sein des patriarches se trouve très éloigné du Christ et du ciel où sont supposés se rendre les élus à la fin des temps. Cette configuration implique par conséquent que le sein d'Abraham a conservé son sens premier.
Les élus dans le sein des patriarches

Singulièrement, les élus regroupés dans le sein des trois patriarches sont vêtus et sexuellement différenciés, ce qui permet de reconnaître des femmes et des laïcs à gauche, des moines et d'autres laïcs au milieu, des évêques, un moine, un simple clerc et à nouveau des laïcs à droite. La hiérarchie s'organise donc logiquement à partir de la droite qui correspond ici à l'entrée du paradis : les évêques d'abord, les femmes à la fin ${ }^{51}$. Ils ne se rattachent pas particulièrement aux scènes de la contre-façade car dix d'entre eux regardent dans cette direction tandis que les neuf autres se tournent vers la gauche. Leur apparence est théoriquement celle des corps ressuscités et non celle des âmes séparées étant donné que celles-ci devraient être indifférenciées comme dans les exemples évoqués précédemment. Elles possèdent cependant la taille des âmes et, plus significativement encore, les élus de Bominaco, dont le statut d'âmes séparées ne fait aucun doute, présentent la même apparence, ce qui permet d'interpréter les élus de Fossa dans le même sens.

Une particularité totalement exceptionnelle vient confirmer qu'il s'agit bien d'âmes séparées et que le premier registre correspond effectivement au jugement immédiat. Un

\footnotetext{
${ }^{49}$ Chorus angelorum te suscipiat [et in sinu Abrahae te collocet], et cum Lazaro quondam paupere aeternam habeas requiem (CAO III, 1782, éd. R.-J. HESBERT, Corpus Antiphonalium Officii, 6 vol., Rome, 1963-1979, III, p. 95). Suscipiat te Christus qui vocavit te, et in sinum Abrahae angeli deducant te (CAO III, 5092, éd. ibid., III, p. 499). Il faut toutefois signaler que dans la Vision d'Albéric de Settefrati (chap. XX et XXXIX), Abraham, Lazare et le bon larron se trouvent dans un paradis accessible uniquement à la fin des temps mais dans le même temps très éloigné de Dieu qui trône dans le septième ciel, cf. M. INGUANEZ et A. MIRRA, La visione di Alberico, in Miscellanea Cassinese, 1932, p. 33-103, en part p. 98 et 99.

${ }^{50}$ C. CONSOLI, Il Giudizio Finale del Battistero di Firenze e il suo pubblico, in Quaderni medievali, 9, 1980, pp. 55-83 ; A. GARZELLI, Per une lettura del Giudizio Universale nel Battistero di Firenze, dans Romanico padano, romanico europeo, Parme, 1982 (Atti del convegno internazionale di studi, ModenaParma, 26 ottobre- $1^{\circ}$ novembre 1977), pp. 399-410 ; A.M. GIUSTI, I mosaici della cupola, in A. PAOLUCCI (éd.), Il Battistero di San Giovanni a Firenze, 2 vol., Modène, 1994 (Mirabilia Italiae, 2), pp. 281-342 et pp. 715-820 ; I. HUECK, Il programma dei Mosaici, in ibid., pp. 229-263 ; et I. GRÖTECKE, Das Bild des Jüngsten Gerichts : die ikonographischen Konventionen in Italien und ihre politische Aktualisierung in Florenz, Worms, 1997.

${ }^{51}$ Aucun évêque ne figure dans le cortège des élus ressuscités, les premiers étant des clercs dépourvus de signes distinctifs. Cette configuration inversée par rapport à la formule byzantine pourrait laisser entendre que le sein des patriarches est un séjour définitif. Les arguments favorables à l'hypothèse du double jugement restent à mes yeux prépondérants, mais l'absence d'évêque dans ce cortège demeure un contre-argument notable.
} 


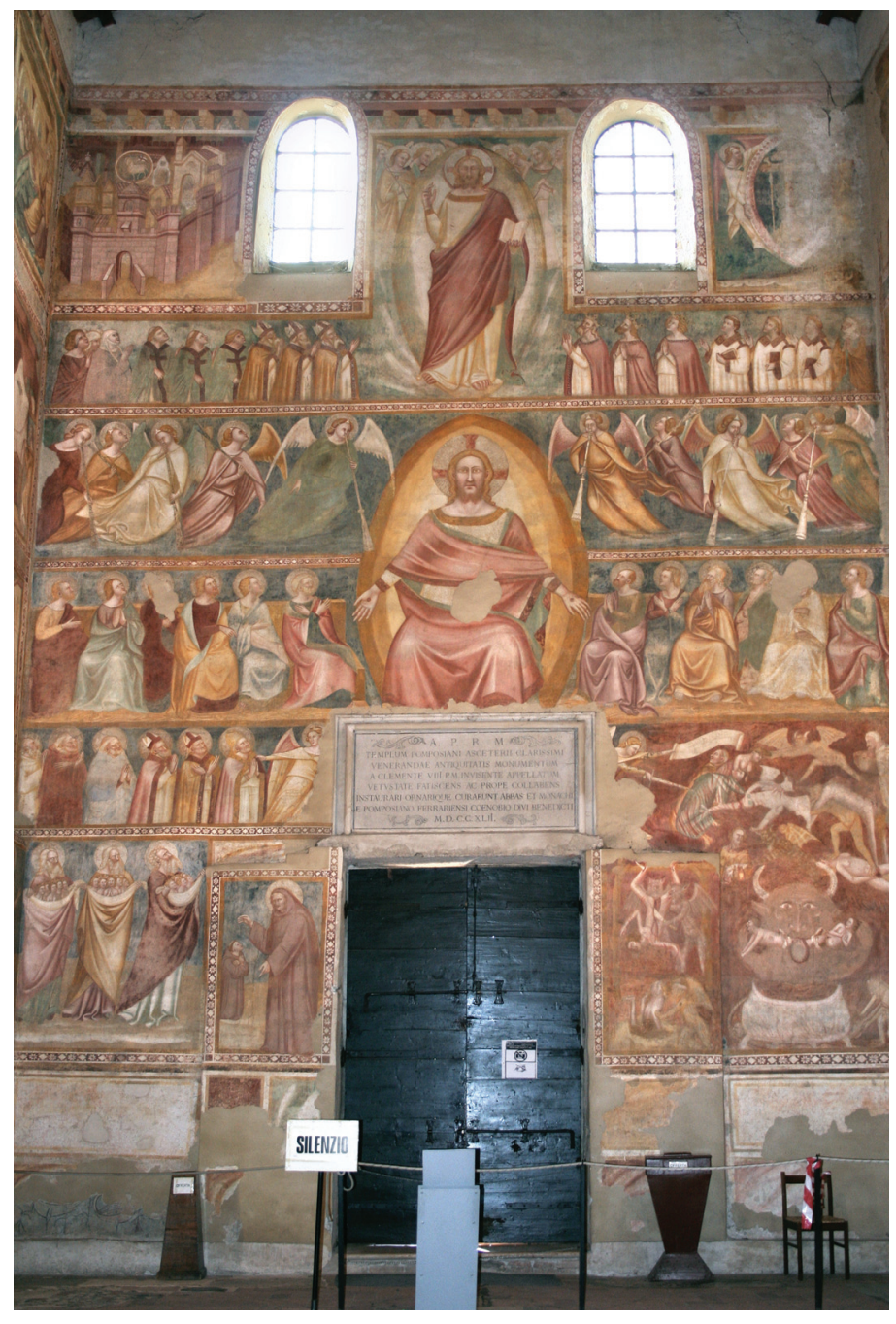

fig. 13 Pomposa, ancienne église abbatiale, contre-façade, le Jugement dernier (photo M. Angheben).

petit personnage vêtu d'une simple tunique blanche surgit derrière les figures de Jacob et d'Abraham en tendant une main vers le haut (fig. 11-12). De toute évidence, ces figures représentent les âmes quittant le paradis d'attente pour rejoindre leur corps ressuscité par le Christ et accéder ensuite au royaume des cieux. Alors que l'iconographie montre généralement les âmes transportées par des anges jusque dans le sein d'Abraham, les peintures de Fossa donnent à voir l'obsolescence de ce séjour et le départ des âmes. De manière éminemment significative, ce détail ne figure pas à Bominaco, ce qui est parfaitement conséquent puisque son paradis s'inscrit dans le temps présent et non au moment où il doit s'effacer au profit du royaume des cieux ${ }^{52}$.

Si cette formule demeure totalement exceptionnelle, l'idée a été exprimée ailleurs sous d'autres formes. À la cathédrale d'Amiens, le patriarche élève les âmes des élus vers le haut, exprimant ainsi que ces âmes doivent désormais le quitter et s'élever vers le ciel. Cette ascension se prolonge d'ailleurs à la deuxième voussure où des chérubins transportent les élus de

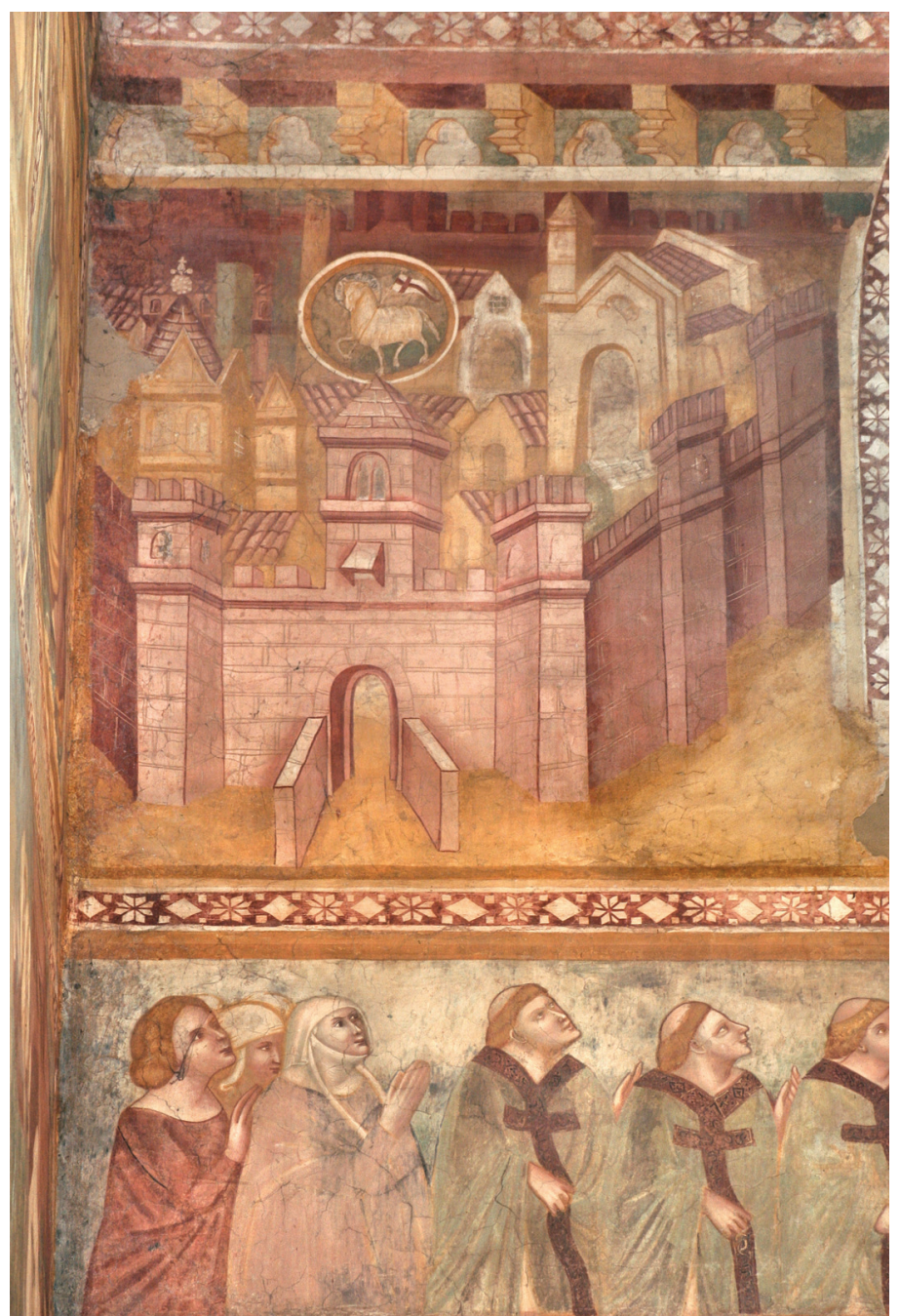

fig. 14 Pomposa, ancienne église abbatiale, contre-façade, la Jérusalem céleste (photo M. Angheben).

telle manière que ceux-ci puissent contempler le Christ du tympan et bénéficier ainsi de la vision béatifique ${ }^{53}$. Le thèmea été repris et développéun siècle plus tard à la contre-façade de

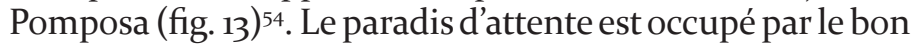
larron et par le sein des patriarches, comme dans la formule byzantine, si ce n'est que les patriarches se tournent vers le haut et que le premier, sans doute Abraham, commence à élever ses protégés qui se tournent également vers le Christ. Dans cette vaste composition, un argument décisif permet de confirmer l'hypothèse de l'abandon de ce séjour : l'angle supérieur gauche est occupé par la Jérusalem céleste dominée par l'Agneau (fig. 14). Il ne fait dès lors aucun doute ici que les élus ressuscités sont destinés non pas au sein des patriarches mais à la Cité sainte.

\section{CONCLUSION}

La plupart des composantes structurelles et iconographiques permettent donc d'interpréter les peintures de

\footnotetext{
${ }_{52}^{2}$ À Santa Maria de Ronzano, le thème des âmes quittant le sein des patriarches a été repris et même amplifié, ce qui laisserait supposer que ces peintures appartenaient initialement à un Jugement dernier, mais rien ne permet de l'affirmer.

${ }_{53}$ W. SCHLINK, Der Beau-Dieu von Amiens. Das Christusbild der gotischen Kathedrale, Francfort et Leipzig, 1991, p. 92 ; et M. ANGHEBEN, D’un Jugement à l'autre..., pp. 584-587.

${ }^{54}$ M.L. GATTI PERRER (éd.), "La dimora di Dio con gli uomini” (Ap 21, 3). Imagini della Gerusalemme celeste dal III al XIV secolo, Milan, 1983, pp. 234-235 ; et A. VOLPE, Pittura a Pomposa, in A. SAMARITANI et C. DI FRANCESCO (éd.), Pomposa. Storia, Arte, Architettura, Ferrare, 1999, pp. 95-149, en part. p. 120.
} 


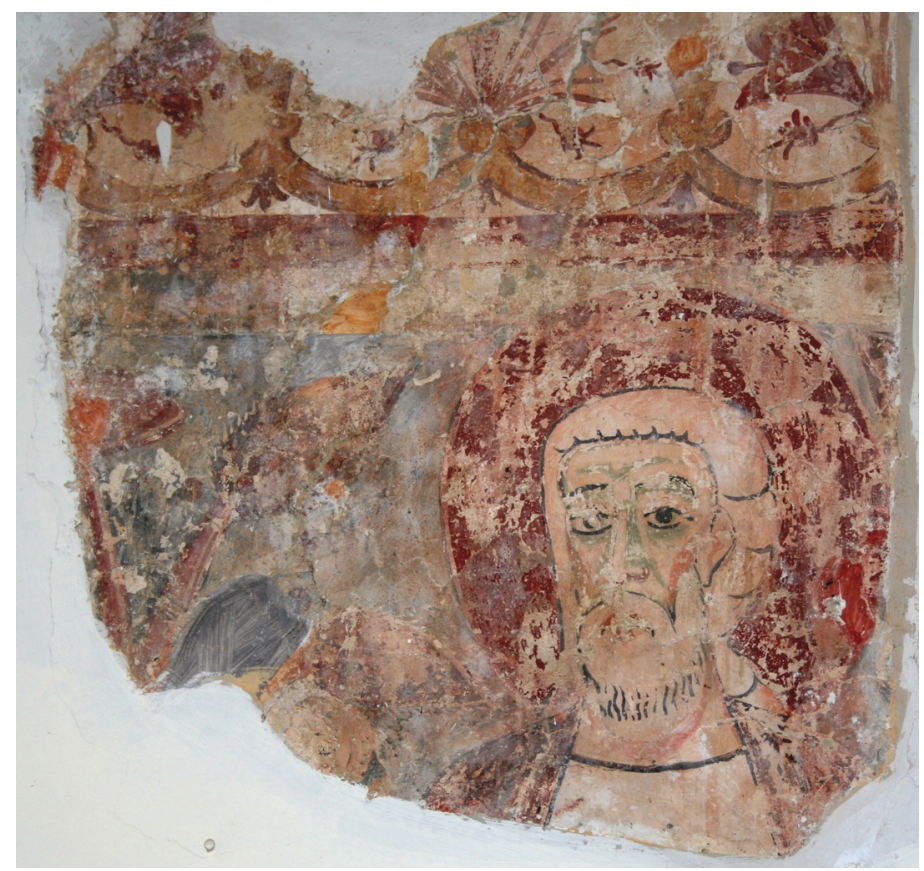

fig. 15 Pescosansonesco Nuovo, Santa Maria Assunta, contre-façade, détail du Jugement dernier, un patriarche et un élu (photo M. Angheben).

Fossa comme une représentation des deux jugements. Cette solution émane naturellement des modèles byzantins, mais elle présente également des traits originaux et extrêmement significatifs comme les âmes quittant le sein des patriarches et l'autel figuré derrière la balance, si cette interprétation est exacte. Dans cette perspective, la composition doit être lue en deux temps. Tout d'abord, les âmes sont jugées une première fois après la mort au moyen d'une balance. Selon le verdict, elles se rendent en enfer ou dans le paradis d'attente, sans doute guidées par la Vierge et peut-être par saint Pierre. Accueillies dans un jardin paradisiaque, une sorte d'Éden céleste, et regroupées dans le sein des patriarches en fonction de leur hiérarchie, elles attendent patiemment la fin des temps. De manière tout à fait exceptionnelle, ce tableau s'inscrit à la fois dans le temps présent et dans le temps futur car deux âmes s'apprêtent déjà à le quitter pour rejoindre leur corps ressuscité. Le regard est ainsi invité à appréhender dans un second temps les événements futurs en considérant à la fois l'apparition du Christ, du tribunal divin et des anges buccinateurs dans le ciel, et la résurrection des morts sur terre. Ce deuxième jugement s'achève par une nouvelle séparation : les damnés sont repoussés vers l'enfer, qui est ici le même que pour les âmes séparées, tandis que les élus sont invités à se rapprocher du Juge et à s'élever vers le ciel, comme le suggère leur gestuelle. Leur destination finale n'est pas représentée, mais c'est généralement le cas dans les Jugements derniers, qu'ils soient occidentaux ou orientaux. Les fresques de Pomposa constituent de ce point de vue une exception notable.

Au départ de cet exemple dont l'interprétation ne soulève à mon sens presque aucun doute, on peut étendre l'hypo-



fig. 16 Sommacampagna, Sant'Andrea, contre-façade, le sein d'Abraham (photo M. Angheben).

thèse à des compositions moins explicites. C'est le cas en particulier pour les peintures de Sant'Angelo in Formis dont certains indices évoqués précédemment corroborent cette lecture. On peut également la soutenir pour les peintures de Santa Maria Assunta de Pescosansonesco Nuovo stylistiquement et iconographiquement très proches de celles de Fossa (fig. 15). Un fragment montre en effet la tête d'un patriarche se détachant sur un fond verdoyant et surtout la tête d'un élu située au niveau des épaules de son protecteur, ce qui permet de supposer qu'il appartient non pas à la catégorie des élus rassemblés dans le sein des patriarches mais à celle des âmes qui commencent à s'en éloigner ${ }^{55}$. Cet élu se dirige cependant vers la gauche et se détourne ainsi du Christ, si bien que ce programme fragmentaire ne peut pas être interprété avec certitude en termes de double jugement ${ }^{56}$.

L'hypothèse peut également être proposée pour les peintures de Sommacampagna où l'un des élus du sein d'Abraham - celui de droite - tourne le regard et les deux mains vers le haut (fig. 16) ${ }^{57}$. Et comme je pense l'avoir montré, elle peut s'appliquer avec certitude au Jugement dernier de Pomposa. Je considère qu'elle peut également être transposée au tondo du Vatican mais cette hypothèse réclame une argumentation

\footnotetext{
55 V. LUCHERINI, Pittura tardoduecentesca..., p. 84 ; P. LEONE DE CASTRIS, Affresco del Giudizio Universale. Chiesa di Santa Maria in Blesiano. Pescosansonesco, in Documenti dell'Abruzzo Teramano, V, 1 Dalla valle del Fino alla valle del medio e basso Pescara, Pescara, 2003, pp. 472-474. Pour cet auteur, le style de ces peintures est plus proche de celui des thèmes « laïcs » de Fossa (saints cavaliers, mois, donateurs).

${ }^{56}$ À Ronzano, trois élus se dirigent également vers la gauche. On ignore cependant si ce programme comportait initialement des composantes deutéroparousiaques, de sorte que l'interprétation de cette particularité iconographique demeure incertaine.

57 F. BUTTURINI, La pittura frescale dell'anno mille nella diocesi di Verona, Vérone, 1987, pp. 48-53.
} 
dépassant amplement le cadre de cet article. La formule byzantine dans laquelle cohabitent les deux jugements a donc été comprise et réinterprétée par les concepteurs de deux Jugements derniers italiens et sans doute davantage.

À Fossa, trois raisons peuvent expliquer le choix du double jugement. D'une part, il correspond aux conceptions théologiques dans lesquelles le sort de l'humanité se joue en deux temps. Il faut toutefois relever que dans la deuxième moitié du XIII ${ }^{\text {e }}$ siècle, on considère qu'il n'existe qu'un seul lieu paradisiaque et que la vision béatifique n'est plus reportée à la fin des temps ${ }^{5}$. Les peintures de Fossa pourraient donc être considérées comme archaïques de ce point de vue. Le milieu dans lequel est née cette composition demeure malheureusement mal connu, peut-être une dépendance de l'abbaye cistercienne d'Ocre ${ }^{59}$, mais on peut difficilement lui attribuer la responsabilité de cet apparent archaïsme puisqu'on le retrouve dans deux abbayes bénédictines Bominaco et Pomposa - et, dans le cas de Pomposa, près d'un siècle plus tard, après la querelle de la vision béatifique déclenchée par Jean XXII et éteinte par son successeur Benoît XII (1334-1342). La prégnance de la tradition iconographique semble donc avoir joué un rôle prépondérant par rapport à l'évolution des conceptions relatives aux séjours de l'au-delà.

La deuxième motivation vient sans doute de ce que les vivants ne peuvent agir en faveur des défunts à travers les suffrages - les prières, les aumônes et surtout le sacrifice eucharistique - qu'entre l'agonie et la fin des temps ${ }^{60}$. Si la pesée de Fossa et celle de Bominaco se déroulaient effectivement devant un autel, cela signifierait que les concepteurs ont voulu mettre en évidence l'indispensable médiation des clercs et, plus globalement, de l'Église dans l'obtention du salut des défunts. Les enjeux institutionnels et économiques liés à l'administration des suffrages étaient en tout cas suffisamment importants pour motiver l'association du jugement immédiat au Jugement dernier.

Il se pourrait enfin que le ou les donateurs aient opté pour cette formule dans le but de se faire représenter séparément du côté des élus tout en évitant d'apparaître dans le cortège des ressuscités, ce qui aurait pu sembler inconvenant dans la mesure où le verdict ultime émane directement de Dieu. Si tel était le cas, cela signifierait que le concepteur a tenu compte à la fois des préoccupations des clercs et de celles des laïcs. On ne peut malheureusement avoir aucune certitude sur l'identité de l'élue marchant vers le paradis et, par conséquent, sur la validité de cette supposition. On peut en tout cas retenir que le concepteur de cette composition - ou de son hypothétique modèle - a su appliquer le schème byzantin des deux jugements tout en l'enrichissant de compléments parfaitement adaptés à cette double thématique.

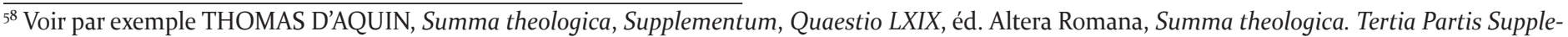
mentum, Rome, 1894, pp. 381-391. Pour le point de vue de Bonaventure, voir C. TROTTMANN, La vision béatifique. Des disputes scolastiques à sa définition par Benoit XII, Rome 1995, pp. 197-208.

59 D. PICCIRILLI, Committenza francese..., p. 53.

${ }^{60}$ Voir notamment C. TREFFORT, L'Église carolingienne et la mort. Christianisme, rites funéraires et pratiques commémoratives, Lyon, 1996, pp. 25 -26 ; et M. LAUWERS, La mémoire des ancêtres, le souci des morts. Morts, rites et société au Moyen Âge (diocèse de Liège, XI ${ }^{e}-X I I I^{e}$ siècles), Paris, 1997, pp. 67-85. On notera que pour saint Bonaventure (Breviloquium 7, 3, éd. QUARACCHI et L. PRUNIÈRES, Saint Bonaventure, Breviloquium 7. Le Jugement dernier, Paris, 1967, pp. 70-79), les suffrages ne concernent que les âmes du purgatoire. Le purgatoire ne pouvait toutefois pas être représenté car son iconographie ne s'était pas encore imposée.
} 